\title{
LIMIT THEOREMS IN FREE PROBABILITY THEORY. II
}

\author{
G. P. CHISTYAKOV ${ }^{1,3}$ AND F. GÖTZE ${ }^{2,4}$
}

\begin{abstract}
Based on an analytical approach to the definition of multiplicative free convolution on probability measures on the nonnegative line $\mathbb{R}_{+}$and on the unit circle $\mathbb{T}$ we prove analogs of limit theorems for nonidentically distributed random variables in classical Probability Theory.
\end{abstract}

\section{INTRODUCTION}

In the last years free convolution of measures introduced by D. Voiculescu has been intensively studied. The key concept of this definition is the notion of freeness, which can be interpreted as a kind of independence for noncommutative random variables. As in the classical probability the concept of independence gives rise to the classical convolution, the concept of freeness leads to a binary operation on the probability measures on the real line, the free convolution. Many classical results in the theory of addition of independent random variables have their counterpart in this new theory, such as the law of large numbers, the central limit theorem, the Lévy-Khintchine formula and others. We refer to Voiculescu, Dykema and Nica [17] for introduction to these topic. Bercovici and Pata [7] established the distributional behavior of sums of free identically distributed random variables and described explicitly the correspondence between classical and free limits. They found remarkable parallelism between the free additive and classical additive infinite divisibility and limits laws for free and classical additive convolution. In the paper [11], using an analytical approach to the definition of the additive free convolution (see [10]), the Bercovici and Pata result was proved in the case of free non-identically distributed random variables, i. e., it was shown that the Bercovici and Pata parallelism holds in the general case of free non-identically distributed random variables. Our approach to

Date: April, 2007.

1991 Mathematics Subject Classification. Primary 46L50, 60E07; Secondary 60E10.

Key words and phrases. Free random variables, Nevanlinna functions, Schur functions, free convolutions, limit theorems.

1) Institute for Low Temperature Physics and Engineering, Kharkov, Ukraine.

2) Faculty of Mathematics, University of Bielefeld, Germany.

3) Research supported by the SFB 701. Partially supported by INTAS grant N 03-51-5018 and by the join Ukraine-French collaboration Dnipro, 2007-2008.

4) Research supported by the SFB 701. Partially supported by INTAS grant N 03-51-5018. 
the definition of additive free convolution allowed us to obtain estimates of a rate of convergence of distribution functions of free sums. We proved a semi-circle approximation theorem (an analog of the Berry-Esseen inequality), a law of large numbers with estimates of convergence. We described the class Lévy $\mathcal{L}_{\boxplus}$ for free random variables and we gave the canonical representation of the measures of the class $\mathcal{L}_{\boxplus}$ and gave a characterization of the class $\mathcal{L}_{\boxplus}$ with the help of the property of self-decomposability, extending results by Barndorff-Nielsen and Thorbjørnsen [3]. In this paper we study the case of free multiplicative convolutions, using again an analytical approach to the definition of free convolutions, and we prove limit theorems for probability measures (p-measures) on $\mathbb{R}_{+}=[0, \infty)$ and on $\mathbb{T}=\{z \in \mathbb{C}:|z|=1\}$ in the case of non-identically distributed p-measures. Our results generalize the Bercovici-Pata result for p-measures on $\mathbb{R}_{+}$in the case of identically distributed p-measures [8] and are new for p-measures on $\mathbb{T}$. We would like to emphasize that our approach allows to obtain explicit estimates of a convergence in these limit theorems. However, we do not address this problem in this paper.

The paper is organized as follows. In Section 2 we formulate and discuss the main results of the paper. In Section 3 we formulate auxiliary results. In Section 4 we prove a multiplicative free limit theorem for p-measures on $\mathbb{R}_{+}$which implies the Bercovici and Pata parallelism between free multiplicative and classical multiplicative infinite divisibility and between limits laws for free and classical multiplicative convolution in the general case of free non-identically distributed random variables. In Section 5 we prove the multiplicative free limit theorem for p-measures on $\mathbb{T}$ and compare this result with limit theorems for p-measures on $\mathbb{T}$ with respect to the operation of the classical convolution.

\section{Results}

Denote by $\mathcal{M}_{+}$the set of p-measures on $\mathbb{R}_{+}=[0,+\infty)$. Let $X$ and $Y$ be free random variables in some noncommutative probability space having distributions $\mu$ and $\nu$ respectively. Then the multiplicative free convolution of $\mu$ and $\nu$, denoted by $\mu \otimes \nu$, is the distribution of $X^{1 / 2} Y X^{1 / 2}$. The p-measures $\mu \otimes \nu$ have been introduced by Voiculescu [16] for compactly supported p-measures and by Bercovici and Voiculescu [6] for the class $\mathcal{M}_{+}$.

Define, following Voiculescu [16], the $\psi_{\mu}$-function of a probability measure $\mu \in \mathcal{M}_{+}$, by

$$
\psi_{\mu}(z)=\int_{\mathbb{R}_{+}} \frac{z u}{1-z u} \mu(d u), \quad z \in \mathbb{C} \backslash \mathbb{R}_{+} .
$$

The measure $\mu$ is completely determined by $\psi_{\mu}$ because $z\left(\psi_{\mu}(z)+1\right)=G_{\mu}(1 / z)$, where

$$
G_{\mu}(z):=\int_{\mathbb{R}} \frac{\mu(d u)}{z-u}, \quad z \in \mathbb{C}^{+}=\{z \in \mathbb{C}: \Im z>0\} .
$$

Note that $\psi_{\mu}: \mathbb{C} \backslash \mathbb{R}_{+} \rightarrow \mathbb{C}$ is an analytic function such that $\psi_{\mu}(\bar{z})=\overline{\psi_{\mu}(z)}$, and $\psi_{\mu}(z) \in \mathbb{C}^{+} \cup \mathbb{R}$ for $z \in \mathbb{C}^{+}$. Introduce the $R_{\mu}$-function of the measure $\mu$ by

$$
R_{\mu}(z):=\psi_{\mu}(z) /\left(1+\psi_{\mu}(z)\right), \quad z \in \mathbb{C} \backslash \mathbb{R}_{+} .
$$


We see that the function $R_{\mu}(z)$ belongs to the class $\mathcal{N}$, i.e., $R_{\mu}(z)$ is analytic such that $R_{\mu}: \mathbb{C}^{+} \rightarrow \mathbb{C}^{+} \cup \mathbb{R}$, and is analytic and non-positive on the negative part of $\mathbb{R}$. Moreover, for $x>0, R_{\mu}(-x) \rightarrow 0$ as $x \rightarrow 0$.

Denote by $\mathcal{K}$ the subclass of $\mathcal{N}$ of functions $f$ such that $f(z) \in \mathcal{N}$ and $f(z) / z \in \mathcal{N}$, and, for $x>0, f(-x) \rightarrow 0$ as $x \rightarrow 0$.

Using the approach to the definition of the multiplicative free convolution in Chistyakov and Götze [10], we define the multiplicative free convolution in the following way.

Let $\mu_{1}$ and $\mu_{2}$ belong to $\mathcal{M}_{+}$and let $R_{\mu_{1}}(z)$ and $R_{\mu_{2}}(z)$ be Nevanlinna functions which correspond to these measures by the relation (2.2). We shall define the free multiplicative convolution based on $R_{\mu_{1}}(z)$ and $R_{\mu_{2}}(z)$ only. The function $R_{\mu}(z)$ is an analogue of the reciprocal Cauchy transform of the measure $\mu \in \mathcal{M}_{+}$.

It was proved [10] that there exist two unique functions $Z_{1}(z)$ and $Z_{2}(z)$ in the class $\mathcal{K}$ such that

$$
Z_{1}(z) Z_{2}(z)=z R_{\mu_{1}}\left(Z_{1}(z)\right) \quad \text { and } \quad R_{\mu_{1}}\left(Z_{1}(z)\right)=R_{\mu_{2}}\left(Z_{2}(z)\right), \quad z \in \mathbb{C}^{+} .
$$

Note (for details see Section 3 ) that $R(z):=R_{\mu_{1}}\left(Z_{1}(z)\right.$ ) belongs to the class $\mathcal{K}$. Introduce the function $\psi(z):=R(z) /(1-R(z))$. We see that $\psi \in \mathcal{K}$ and thus conclude (see again Section 3) that $\psi(z)$ admits the representation (2.1) with some p-measure $\mu \in \mathcal{M}_{+}$, that is $\psi(z)=\psi_{\mu}(z), z \in \mathbb{C}^{+}$. In addition $R(z)=R_{\mu}(z), z \in \mathbb{C}^{+}$. Therefore $\psi_{\mu_{1}}\left(Z_{1}(z)\right)=\psi_{\mu}(z)$.

The measure $\mu$ is determined in a unique way by the measures $\mu_{1}$ and $\mu_{2}$. We write $\mu:=\mu_{1} \otimes \mu_{2}$.

In this way the multiplicative free convolution of p-measures on $\mathbb{R}_{+}$is defined by complex analytic methods. This has been proved independently by different means in [4].

The existence and uniqueness of subordinating functions $Z_{j}(z)$ in (2.3) has been studied earlier using other methods in [9], [18]-[20].

The function $R_{\mu}(z)$ is univalent on the left half-plane $i \mathbb{C}^{+}$(see [6]). Let $\tilde{\chi}_{\mu}$ be the right inverse of this function on the image $R_{\mu}\left(i \mathbb{C}^{+}\right)$. We define the $\Sigma$-transform of $\mu$ as the function $\Sigma_{\mu}(z):=\tilde{\chi}_{\mu}(z) / z$ defined on $R_{\mu}\left(i \mathbb{C}^{+}\right)$.

From (2.3) we conclude that the relation

$$
\frac{R_{\mu_{1}}^{(-1)}(z)}{z} \frac{R_{\mu_{2}}^{(-1)}(z)}{z}=\frac{R_{\mu}^{(-1)}(z)}{z} \quad \text { or } \quad \Sigma_{\mu_{1}}(z) \Sigma_{\mu_{2}}(z)=\Sigma_{\mu}(z)
$$

holds on a domain where all functions $\Sigma_{\mu_{1}}, \Sigma_{\mu_{2}}$ and $\Sigma_{\mu}$ are defined. This formula was first proved by Voiculescu [16] (see also [6]). From (2.4) we deduce that our definition of the multiplicative convolution $\mu_{1} \otimes \mu_{2}$ coincides with Voiculescu's definition.

We now introduce the notion of infinitely divisible measures for multiplicative free convolution. More precisely, a measure $\mu \in \mathcal{M}_{+}$is said to be $\nabla$-infinitely divisible if for every natural number $n$ there exists a measure $\nu_{n} \in \mathcal{M}_{+}$such that

$$
\mu=\nu_{n} \otimes \nu_{n} \otimes \cdots \otimes \nu_{n}(n \text { times })
$$


with $\nu_{n} \in \mathcal{M}_{+}$. These measures were studied intensively in Voiculescu [16], Bercovici and Voiculescu [5], [6]. There is an analogue of the Lévy-Khintchine formula which states that a measure $\mu \in \mathcal{M}_{+}$is $\otimes$-infinitely divisible if and only if there exist a finite nonnegative measure $\sigma$ on $[0, \infty)$ and real numbers $a$ and $b \geq 0$ such that

$$
\Sigma_{\mu}(z)=\exp \left\{a+\frac{b}{z}-\int_{\mathbb{R}_{+}} \frac{u+z}{1-u z} \sigma(d u)\right\} .
$$

Since there is a one-to-one correspondence between the functions $\Sigma_{\mu}(z)$ and the triples $(a, b, \sigma)$, we shall write $\Sigma_{\mu}=(a, b, \sigma)$.

In other words, a measure $\mu \in \mathcal{M}_{+}$is $\bigotimes$-infinitely divisible if and only if

$$
\Sigma_{\mu}(z)=\exp \{-u(z)\}
$$

where $u(z) \in \mathcal{N}$ and $u(z)$ is analytic and real-valued on the negative part of $\mathbb{R}$.

As in the case of additive free convolution we can formulate the limit problem for multiplicative free convolution. Let $\left\{\mu_{n k}: n \geq 1,1 \leq k \leq k_{n}\right\}, k_{n} \uparrow \infty$ as $n \rightarrow \infty$, be a triangular scheme of measures in $\mathcal{M}_{+}$such that

$$
\lim _{n \rightarrow \infty} \max _{1 \leq k \leq k_{n}} \mu_{n k}(\{u:|u-1|>\varepsilon\})=0
$$

for every $\varepsilon>0$, and let $\left\{a_{n}: n \geq 1\right\}$ be a sequence of positive numbers. The measures $\mu_{n k} \in \mathcal{M}_{+}$are called infinitesimal. Denote by $\delta_{a}$ a p-measure such that $\delta_{a}(\{a\})=1$. We would like to

1) determine all $\mu \in \mathcal{M}_{+}$such that $\mu^{(n)}=\delta_{1 / a_{n}} \otimes \mu_{n 1} \otimes \mu_{n 2} \cdots \otimes \mu_{n k_{n}} \rightarrow \mu$ in the weak topology;

2) determine conditions such that $\mu^{(n)}$ converges weakly to a given $\mu$.

For measures $\mu_{n k} \in \mathcal{M}_{+}$we denote by $\widehat{\mu}_{n k}$ the measures defined by $\widehat{\mu}_{n k}((-\infty, x)):=$ $\mu_{n k}\left(\left(-\infty, a_{n k} x\right)\right), x>0$, where $a_{n k}:=\int_{(1-d, 1+d)} u \mu_{n k}(d u)$. Here $d \in(0,1)$ is an arbitrary fixed number.

We shall give a complete solution of this problem, proving a multiplicative free Limit Theorem for measures in $\mathcal{M}_{+}$.

Theorem 2.1. Let $\mu_{n k}$ be a triangular scheme of infinitesimal probability measures. Then we have

(a) The family of limit measures in the weak topology of sequences $\mu^{(n)}=\delta_{1 / a_{n}} \otimes \mu_{n 1} \otimes$ $\mu_{n 2} \otimes \cdots \otimes \mu_{n k_{n}}$ coincides with the family of $\bigotimes$-infinitely divisible measures.

(b) There exist positive constants $a_{n}$ such that the sequence $\mu^{(n)}=\delta_{1 / a_{n}} \otimes \mu_{n 1} \otimes \mu_{n 2} \otimes$ $\ldots \otimes \mu_{n k_{n}}$ converges weakly if, and only if, $\sigma_{n}$ converges weakly on $[0, \infty]$ to some finite nonnegative measure $\sigma$ on $[0, \infty]$, where, for any Borel set $S \subseteq[0, \infty]$,

$$
\sigma_{n}(S):=\sum_{k=1}^{k_{n}} \int_{S} \frac{(u-1)^{2}}{1+u^{2}} \widehat{\mu}_{n k}(d u) .
$$


Then all admissible $a_{n}$ are of the form $a_{n}=\exp \left\{\alpha_{n}-\alpha+o(1)\right\}$, where $\alpha$ is an arbitrary finite number and

$$
\alpha_{n}=\sum_{k=1}^{k_{n}}\left(\log a_{n k}+\int_{\mathbb{R}_{+}} \frac{u^{2}-1}{1+u^{2}} \widehat{\mu}_{n k}(d u)\right),
$$

and all possible limit measures $\mu \in \mathcal{M}_{+}$have a Voiculescu transform $\Sigma_{\mu}=(\alpha$, $\sigma(\{\infty\}), \sigma)$.

Theorem 2.1 was proved by Bercovici and Pata [8] in the identical case $\mu_{n 1}=\cdots=\mu_{n k_{n}}$.

Denote by $\mathcal{M}_{+}^{0}$ the set of p-measures on $(0, \infty)$. The classical multiplicative convolutions of two measures $\mu, \nu$ on $(0, \infty)$ is denoted by $\mu \circledast \nu$. Thus, $\mu \circledast \nu$ is the probability distribution of $X Y$, where $X$ and $Y$ are classical (commuting) independent random variables with probability distributions $\mu$ and $\nu$ respectively. The study of $\circledast$-infinitely divisible p-measures reduces (by a change of variable) to the study of the usual infinitely divisible measures on $\mathbb{R}$. The Fourier transform needs to be replaced by the Mellin transform of a measure $\mu$ on $(0, \infty)$ defined by

$$
M_{\mu}(t)=\int_{(0, \infty)} u^{i t} \mu(d u), \quad t \in \mathbb{R}
$$

We have

$$
M_{\mu \circledast \nu}(t)=M_{\mu}(t) M_{\nu}(t), \quad t \in \mathbb{R} .
$$

Moreover the classical Lévy-Khinchin formula is as follows (see [12], [13] for the additive case and [8]):

A p-measure $\mu$ on $(0, \infty)$ is $\circledast$-infinitely divisible if and only if there exist a finite nonnegative Borel measure $\sigma$ on $(0, \infty)$ and a real number $a$ such that

$$
M_{\mu}(t)=\exp \left\{m_{\mu}(t)\right\}=\exp \left\{i a t+\int_{(0, \infty)}\left(u^{i t}-1-\frac{i t \log u}{1+\log ^{2} u}\right) \frac{1+\log ^{2} u}{\log ^{2} u} \sigma(d u), \quad t \in \mathbb{R} .\right.
$$

Since there is a one-to-one correspondence between functions $M_{\mu}(t)$ and pairs $(a, \sigma)$, we shall write $m_{\mu}=\{a, \sigma\}$.

Comparing the formulation of Theorem 2.1 and the formulation of the classical Limit Theorem (see Theorem 3.6 in Section 3, [13], p. 310, [12]), we obtain the following result, which generalizes the corresponding result in [8] for the case $\mu_{n 1}=\cdots=\mu_{n k_{n}}, n \geq 1$.

Theorem 2.2. Let $\mu_{n k}$ be a triangular scheme of infinitesimal probability measures on $(0, \infty)$. The following assertion are equivalent:

(i) The sequence $\mu^{(n)}=\delta_{1 / a_{n}} \circledast \mu_{n 1} \circledast \mu_{n 2} \circledast \cdots \circledast \mu_{n k_{n}}$ converges weakly to $\mu^{\circledast}$ such that $m_{\mu^{\circledast}}=\left\{a^{\circledast}, \sigma^{\circledast}\right\}$.

(ii) The sequence $\mu^{(n)}=\delta_{1 / a_{n}} \otimes \mu_{n 1} \otimes \mu_{n 2} \otimes \cdots \otimes \mu_{n k_{n}}$ converges weakly to $\mu^{\bigotimes}$ such that $\Sigma_{\mu^{凶}}=\left(a^{\bigotimes}, 0, \sigma^{\bigotimes}\right)$, and $\sigma^{\otimes}(\{0\})=\sigma^{\otimes}(\{\infty\})=0$. 
If the equivalent conditions $(i)$ and $(i i)$ are satisfied then the measures $\sigma^{\circledast}$ and $\sigma^{\otimes}$ are related by

$$
\sigma^{\bowtie}(d u)=\frac{1+\log ^{2} u}{\log ^{2} u} \cdot \frac{(u-1)^{2}}{1+u^{2}} \sigma^{\circledast}(d u)
$$

while

$$
a^{\bigotimes}+a^{\circledast}=\int_{(0, \infty)}\left(\frac{u^{2}-1}{u^{2}+1}-\frac{\log u}{1+\log ^{2} u}\right) \frac{1+\log ^{2} u}{\log ^{2} u} \sigma^{\bigotimes}(d u) .
$$

As shown in [8] there exists a sequence $\left\{\mu_{n}\right\}_{n=1}^{\infty}$ from the class $\mathcal{M}_{+}^{0}$ such that $\mu_{n} \otimes$ $\cdots \otimes \mu_{n}$ ( $n$ times $)$ converges weakly, but $\mu_{n} \circledast \cdots \circledast \mu_{n}$ ( $n$ times $)$ does not.

Given two unitary elements $U_{1}, U_{2}$, which are free in some non-commutative probability space $(\mathcal{A}, \varphi)$, we can form their product, which is again a unitary element. The distributions of $U_{1}$ and $U_{2}$ are probability measures, say $\mu_{1}$ and $\mu_{2}$, on the set $\mathcal{T}$ of complex numbers of modulus one. The distribution of $U_{1} U_{2}$ is $\mu_{1} \otimes \mu_{2}$, the multiplicative free convolution of the measures $\mu_{1}$ and $\mu_{2}$.

Let $\mu$ be a probability measure on the unit circle $\mathbb{T}$. We assume that $\mathbb{T}=y:-\pi \leq$ $y<\pi$. Addition in $\mathbb{T}$ is modulo $2 \pi$. Following Voiculescu [16], we define the $\psi$-function of a probability measure $\mu$ on $\mathbb{T}$, by

$$
\psi_{\mu}(z)=\int_{[-\pi, \pi)} \frac{z e^{i y}}{1-z e^{i y}} \mu(d y) .
$$

This is a convergent power series in $\mathbb{D}=\{z \in \mathbb{C}:|z|<1\}$, the open unit disk of $\mathbb{C}$, such that $\psi_{\mu}(0)=0$. Let $\mathcal{M}_{*}$ be the set of p-measures on $\mathbb{T}$ such that $\int_{[-\pi, \pi)} e^{i y} \mu(d y) \neq$ 0 . If $\mu \in \mathcal{M}_{*}$, the function $Q_{\mu}:=\psi_{\mu} /\left(1+\psi_{\mu}\right)$ has a right inverse $Q_{\mu}^{(-1)}$, defined in a neighborhood of 0 , such that $Q_{\mu}^{(-1)}(0)=0$, and we let $\Sigma_{\mu}(z)=Q_{\mu}^{(-1)}(z) / z$ be $\Sigma_{\mu^{-}}$ transform of $\mu$.

Note that

$$
Q_{\mu}(z)=\frac{\psi_{\mu}(z)}{1+\psi_{\mu}(z)}=\frac{F_{\mu}(z)-1}{F_{\mu}(z)+1}
$$

where $F_{\mu}(z):=2 \psi_{\mu}(z)+1$ is a function of Carathéodory's class $\mathcal{C}$. This means (see Section 3) that $F_{\mu}(z)$ is analytic and $F_{\mu}(z): \mathbb{D} \rightarrow-i\left(\mathbb{C}^{+} \cup \mathbb{R}\right)$. Since $F_{\mu}(0)=1$, such functions $F_{\mu}(z)$ (see Section 3) have the form

$$
F_{\mu}(z)=\int_{[-\pi, \pi)} \frac{e^{i y}+z}{e^{i y}-z} \sigma(d y)
$$

where $\sigma$ is a p-measure. We see from $(2.10)$, that $Q_{\mu} \in \mathcal{S}$ and $Q_{\mu}(0)=0, Q_{\mu}^{\prime}(0) \neq 0$, where $\mathcal{S}$ is the class of Schur's functions. By definition (see Section 3) the class $\mathcal{S}$ is the set of analytic functions $\mathbb{D} \rightarrow \overline{\mathbb{D}}$, where $\overline{\mathbb{D}}$ is the closure of $\mathbb{D}$. In the sequel we denote by $\mathcal{S}_{*}$ the subclass of $\mathcal{S}$ which consists of Schur functions $Q_{\mu}$ such that $Q_{\mu}(0)=0$ and 
$Q_{\mu}^{\prime}(0) \neq 0$. Since $Q_{\mu} \in \mathcal{S}$, by the Schwarz lemma, we have $\left|Q_{\mu}(z) / z\right| \leq 1$ for $z \in \mathbb{D}$. Hence $\left|Q^{(-1)}(z) / z\right| \geq 1$ in some neighborhood of 0 .

Let $\mu_{1}$ and $\mu_{2}$ belong to $\mathcal{M}_{*}$ and let $Q_{\mu_{1}}$ and $Q_{\mu_{2}}$ be Schur functions which correspond to these measures, by (2.10). Let us define the free multiplicative convolution $\mu_{1} \otimes \mu_{2}$ based on $Q_{\mu_{1}}$ and $Q_{\mu_{2}}$.

In [10] it was proved that there exist two unique functions $Z_{1}(z)$ and $Z_{2}(z)$ of the class $\mathcal{S}_{*}$ such that

$$
Z_{1}(z) Z_{2}(z)=z Q_{\mu_{1}}\left(Z_{1}(z)\right) \quad \text { and } \quad Q_{\mu_{1}}\left(Z_{1}(z)\right)=Q_{\mu_{2}}\left(Z_{2}(z)\right), \quad z \in \mathbb{D} .
$$

Now we shall consider the function $Q_{\mu_{1}}\left(Z_{1}(z)\right)$. It is easy to see that this function belongs to the Schur class $\mathcal{S}$ and $Q_{\mu_{1}}\left(Z_{1}(0)\right)=0, Q_{\mu_{1}}^{\prime}(0) Z_{1}^{\prime}(0) \neq 0$. Therefore $Q_{\mu_{1}}\left(Z_{1}(z)\right)=Q_{\mu}(z)$ for $z \in \mathbb{D}$, where $Q_{\mu}(z)$ has the form (2.10) for some measure $\mu \in \mathcal{M}_{*}$. This measure is determined in a unique way by the measures $\mu_{1}$ and $\mu_{2}$. We define $\mu:=\mu_{1} \otimes \mu_{2}$.

Thus, the multiplicative free convolution of measures in $\mathcal{M}_{*}$ is defined by complex analytic methods. This has been proved independently by different means in [4]

By the relation (2.10) between the function $Q_{\mu} \in S$ and the function $\psi_{\mu}(z)$, we conclude that $\psi_{\mu}(z)=\psi_{\mu_{1}}\left(Z_{1}(z)\right)$ for $z \in \mathbb{D}$. In addition we have in some neighborhood of 0

$$
\frac{Q_{\mu_{1}}^{(-1)}(z)}{z} \frac{Q_{\mu_{2}}^{(-1)}(z)}{z}=\frac{Q_{\mu}^{(-1)}(z)}{z} \text { or } \quad \Sigma_{\mu_{1}}(z) \Sigma_{\mu_{2}}(z)=\Sigma_{\mu}(z) .
$$

This formula is due to Voiculescu [16].

Infinitely divisible measures for multiplicative free convolution in the case of measures $\mu \in \mathcal{M}_{*}$ satisfy (2.5), where $\mu$ and $\nu_{n}$ belong to $\mathcal{M}_{*}$. The infinitely divisible measures have been intensively studied in Voiculescu [16], Bercovici and Voiculescu [5], [6]. There is an analogue of the Lévy-Khintchine formula which states that a measure $\mu \in \mathcal{M}_{*}$ is $\bigotimes$-infinitely divisible if and only if there exist a finite nonnegative measure $\sigma$ on $\mathbb{T}$ and a real number $a$ such that

$$
\Sigma_{\mu}(z)=\exp \left\{i a-\int_{[-\pi, \pi)} \frac{1+z e^{i y}}{1-z e^{i y}} \sigma(d y)\right\} .
$$

Since there is a one-to-one correspondence between functions $\Sigma_{\mu}(z)$ and pairs $(a, \sigma)$, we shall write $\Sigma=[a, \sigma]$.

In other words, a measure $\mu \in \mathcal{M}_{+}$is $\nabla$-infinitely divisible if and only if

$$
\Sigma_{\mu}(z)=\exp \{-v(z)\}
$$

where $v(z) \in \mathcal{C}$.

Let us formulate the limit problem for multiplicative free convolution in the case of measures $\mu \in \mathcal{M}_{*}$. 
Let $\left\{\mu_{n k}: n \geq 1,1 \leq k \leq k_{n}\right\}, k_{n} \uparrow \infty$ as $n \rightarrow \infty$, be a triangular scheme of measures in $\mathcal{M}_{*}$. We shall say that the measures $\mu_{n k}$ are infinitesimal if

$$
\lim _{n \rightarrow \infty} \max _{1 \leq k \leq k_{n}} \mu_{n k}(\{y:|y|>\varepsilon\})=0
$$

for every $\varepsilon>0$. Let $\left\{a_{n}: n \geq 1\right\}$ be a sequence of numbers of $[-\pi, \pi)$. The limit problem for multiplicative free convolution for measures $\mu \in \mathcal{M}_{*}$ has the same form as for the case of measures $\mu \in \mathcal{M}_{+}$.

Denote by $\widehat{\mu}_{n k}$ the p-measure $\mu_{n k} \otimes \delta_{-a_{n k}}$, where $a_{n k}:=\int_{[-\pi, \pi)} g(y) \mu_{n k}(d y), \quad \bmod 2 \pi$, where $g(y)$ is a bounded continuous function on $[-\pi, \pi)$ such that $g(y)=y$ in a neighborhood of $y=0$ and $g(-y)=-g(y), g(-\pi)=g(\pi-0)$.

We give a complete solution of the limit problem for measures from the class $\mathcal{M}_{*}$, proving the following limit theorem for product of unitary free random variables.

Theorem 2.3. Let $\mu_{n k}$ be a triangular scheme of infinitesimal measures. We have

1. The family of limit measures in the weak topology of sequences $\delta_{-a_{n}} \otimes \mu_{n 1} \otimes \cdots \otimes \mu_{n k_{n}}$ coincides with the family of infinitely divisible distributions.

2. There exist constants $a_{n}$ such that the sequence $\delta_{-a_{n}} \otimes \mu_{n 1} \otimes \cdots \otimes \mu_{n k_{n}}$ converges to $\mu \in \mathcal{M}_{*}$ if, and only if, $\nu_{n}$ converges weakly to some finite nonnegative measure $\nu$, where for every Borel set $S \subset[-\pi, \pi)$,

$$
\nu_{n}(S):=\sum_{k=1}^{k_{n}} \int_{S}(1-\cos y) \widehat{\mu}_{n k}(d y) .
$$

Then all admissible $a_{n}$ are of the form $a_{n}=\alpha_{n}-\alpha+o(1), \bmod 2 \pi$, where $\alpha \in[-\pi, \pi)$ is an arbitrary number, and

$$
\alpha_{n}=\sum_{k=1}^{k_{n}} a_{n k}+\sum_{k=1}^{k_{n}} \int_{[-\pi, \pi)} \sin y \widehat{\mu}_{n k}(d y),
$$

and all possible limit measures $\mu \in \mathcal{M}_{*}$ have a Voiculescu transform of type $\Sigma_{\mu}(z)=[\alpha, \nu]$.

We compare Theorem 2.3 with limit theorems for sums of independent random variables with values on $\mathbb{T}$ (see [14], [15]).

As before $\mathbb{T}$ denotes the group of rotations of the unit circle and the character group $\widehat{\mathbb{T}}$ of the group $\mathbb{T}$ is $\mathbb{Z}$ - the additive group of all integers (with the discrete topology). The characteristic function $q_{\mu}$ of the p-measure $\mu$ on the circle group $\mathbb{T}$ is the function on $\mathbb{Z}$ defined by

$$
q_{\mu}(m)=\int_{[-\pi, \pi)} e^{i m y} \mu(d y), \quad m \in \mathbb{Z} .
$$

Let $\mu_{1} * \mu_{2}$ is the convolution of p-measures on $\mathbb{T}$. We have

$$
q_{\mu_{1} * \mu_{2}}(m)=q_{\mu_{1}}(m) q_{\mu_{2}}(m), \quad m \in \mathbb{Z} .
$$


Recall that a p-measure $\mu$ on $\mathbb{T}$ is said to be idempotent if $\mu * \mu=\mu$. A p-measure $\mu$ is a divisor of a p-measure $\lambda$ if $\lambda=\mu * \nu$ for some p-measure $\nu$.

Note as well that $\mu \otimes \delta_{a}=\mu * \delta_{a}$ for $\mu \in \mathcal{M}_{*}$ and $a \in[-\pi, \pi)$. In addition, as it is easy to see, the measures $\mu \in \mathcal{M}_{*}$ and $\mu \neq \delta_{0}$ are not idempotent.

Defining infinitely divisible p-measures on $\mathbb{T}$ in the usual way, the classical LévyKhinchin formula has the following form (see [15]).

If $\mu$ is an infinitely divisible probability measure without idempotent factors, then $q_{\mu}$ has a representation

$$
q_{\mu}(m)=e^{i a m} \exp \left\{\int_{[-\pi, \pi)}\left(e^{i m y}-1-i m g(y)\right) \frac{\nu(d y)}{1-\cos y}\right\} \quad m \in \mathbb{Z},
$$

where $a \in[-\pi, \pi), \gamma \geq 0, g(\xi)$ is a bounded continuous function on $[-\pi, \pi)$ such that $g(y)=y$ in a neighborhood of $y=0$ and $g(-y)=-g(y), g(-\pi)=g(\pi-0)$ (which is independent of $\mu), \nu$ is a finite nonnegative Borel measure on $\mathbb{T}$. In addition, $\left(e^{i m y}-1-\right.$ $i m g(y)) /(1-\cos y)$ is defined as $-m^{2}$ when $y=0$.

In the following we shall write $q_{\mu}=\langle a, \nu\rangle$, taking into account that $q_{\mu}$ admits the representation (2.19) which is not unique (see Proposition 3.11). But it turns out that if $\langle a, \nu\rangle$ and $\left\langle a^{\prime}, \nu^{\prime}\right\rangle$ are two representations of $q_{\mu}$, then $\nu(\{0\})=\nu^{\prime}(\{0\})$.

Theorem 2.4. Let $\left\{\mu_{n k}\right\}$ be a triangular scheme of infinitesimal p-measures of the class $\mathcal{M}_{*}$. If the sequence $\mu_{n 1} \otimes \cdots \otimes \mu_{n k_{n}}$ converges weakly to a p-measure $\mu^{\square}$ such that

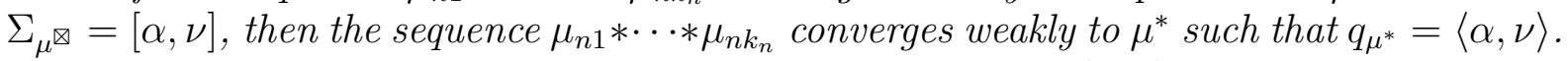
There exists a triangular scheme of infinitesimal p-measures $\left\{\mu_{n k}\right\}$ with $k_{n}=n$ such that the sequence $\mu_{n 1} * \cdots * \mu_{n n}$ of $p$-measures on $\mathbb{T}$ converges weakly to some $p$-measure $\mu \in \mathcal{M}_{*}$ and $\delta_{-a_{n}} \otimes \mu_{n 1} \otimes \cdots \otimes \mu_{n n}$ does not converge weakly for any $a_{n} \in \mathbb{T}$ to a p-measure in $\mathcal{M}_{*}$.

\section{Auxiliary Results}

We need results about some classes of analytic functions (see [1], Section 3, and [2], Section $6, \S 59)$.

The class $\mathcal{N}$ (Nevanlinna, R.) is the class of analytic functions $f(z): \mathbb{C}^{+} \rightarrow\{z: \Im z \geq$ $0\}$. For such functions there is the integral representation

$$
f(z)=a+b z+\int_{\mathbb{R}} \frac{1+u z}{u-z} \tau(d u)
$$

where $b \geq 0, a \in \mathbb{R}$, and $\tau$ is nonnegative bounded measure. Moreover, $a=\Re f(i)$ and $\tau(\mathbb{R})=\Im f(i)-b$.

The Stieltjes-Perron inversion formula for the functions $f$ of the class $\mathcal{N}$ has the following form. 
Let $\rho(u):=\int_{0}^{u}\left(1+t^{2}\right) \tau(d t)$. Then

$$
\rho\left(u_{2}\right)-\rho\left(u_{1}\right)=\lim _{\eta \rightarrow 0} \frac{1}{\pi} \int_{u_{1}}^{u_{2}} \Im f(\xi+i \eta) d \xi,
$$

where $u_{1}<u_{2}$ are continuity points of the function $\rho(u)$.

The function $f(z)$ of the special form

$$
f(z)=\int_{(0, \infty)} \frac{1+u z}{u-z} \tau(d u)
$$

admits the bound

$$
|f(z)|=o(|z|) \quad \text { as } \quad z \rightarrow 0 \quad \text { or } \quad z \rightarrow \infty
$$

nontangentially to $\mathbb{R}$ (i.e., such that $\Re z / \Im z$ stays bounded).

By Krein's results (see [1], and [10], Section 3), the function $R(z) \in \mathcal{K}$ if and only if it admits the following representation

$$
R(z)=b z+z \int_{(0, \infty)} \frac{\tau(d u)}{u-z}, \quad 0<\arg z<2 \pi,
$$

where $b \geq 0$ and $\tau$ is a nonnegative measure such that

$$
\int_{(0, \infty)} \frac{\tau(d u)}{1+u}<\infty
$$

Let $\mu_{j} \in \mathcal{M}, j=1,2$. Recall that we defined $\mu_{1} \otimes \mu_{2}$ in the following way.

Using (2.3) and (3.4), we see that $R(z):=R_{\mu_{1}}\left(Z_{1}(z)\right)$ and $R(z) / z$ belong to the class $\mathcal{N}$ and in addition, for $x>0, R(-x) \rightarrow 0$ as $x \rightarrow 0$. Introduce the function $\psi(z):=$ $R(z) /(1-R(z))$. We note that $\psi(z) \in \mathcal{N}$ and $\psi(z) / z \in \mathcal{N}$. Moreover $\lim _{x \rightarrow-\infty} \psi(x) / x$ $=0$. Hence the function $\psi(z)$ admits the representation (3.4) with $b=0$.

It is easy to see that $\lim _{x \rightarrow-\infty} \psi(x)=-1$ if and only if in the representation (3.4) for $R_{\mu_{1}}(z)$ either $b>0$ or $\tau\left(\mathbb{R}^{+}\right)=\infty$. In this case we obtain for $\psi(z)$ the representation $(2.1)$ with some probability measure $\mu \in \mathcal{M}_{+}$and $\mu(\{0\})=0$, that is $\psi(z)=\psi_{\mu}(z), z \in \mathbb{C}^{+}$. In addition $R(z)=R_{\mu}(z), z \in \mathbb{C}^{+}$. Therefore $\psi_{\mu_{1}}\left(Z_{1}(z)\right)=\psi_{\mu}(z)$.

Let in $(3.4) b=0$ and $\tau\left(\mathbb{R}_{+}\right)<\infty$. Then $\lim _{x \rightarrow-\infty} \psi(x)=-p=-\tau\left(\mathbb{R}_{+}\right) /\left(1+\tau\left(\mathbb{R}_{+}\right)\right)$, and we get for $\psi(z)$ the representation (2.1) with some probability measure $\mu \in \mathcal{M}_{+}$and $\mu(\{0\})=1-p$. Thus, $\psi(z)=\psi_{\mu}(z), z \in \mathbb{C}^{+}$.

The measure $\mu$ is determined in a unique way by the measures $\mu_{1}$ and $\mu_{2}$ and we define $\mu:=\mu_{1} \otimes \mu_{2}$.

As was proved in [10], (2.3) admits the following consequences.

Proposition 3.1. Let $\mu_{1}, \ldots, \mu_{n} \in \mathcal{M}_{+}$. There exist unique functions $Z_{1}(z), \ldots, Z_{n}(z)$ of the class $\mathcal{K}$ such that, for $z \in \mathbb{C}^{+}$,

$$
Z_{1}(z) \ldots Z_{n}(z)=z\left(R_{\mu_{1}}\left(Z_{1}(z)\right)\right)^{n-1}, \quad \text { and } \quad R_{\mu_{1}}\left(Z_{1}(z)\right)=\cdots=R_{\mu_{n}}\left(Z_{n}(z)\right) .
$$


Moreover, $R_{\mu_{1} \otimes \cdots \boxminus \mu_{n}}(z)=R_{\mu_{1}}\left(Z_{1}(z)\right)$ for all $z \in \mathbb{C}^{+}$.

We need the following auxiliary results. The first three of them are due to Bercovici and Voiculescu [6].

Proposition 3.2. Let $\left\{\mu_{n}\right\}_{n=1}^{\infty}$ and $\left\{\nu_{n}\right\}_{n=1}^{\infty}$ be sequences of $p$-measures on $\mathbb{R}_{+}$which converge weakly to $p$-measures $\mu$ and $\nu$, respectively. Then $\left\{\mu_{n} \otimes \nu\right\}_{n=1}^{\infty}$ converges weakly to the $p$-measure $\mu \otimes \nu$. The same result holds for $p$-measures $\mu \in \mathcal{S}_{*}$ on $\mathbb{T}$.

Proposition 3.3. Let $\mu_{1}$ and $\mu_{2}$ be p-measures on $\mathbb{R}_{+}$, and let $\mu=\mu_{1} \otimes \mu_{2}$. Then we have $\mu(\{0\})=\max \left\{\mu_{1}(\{0\}), \mu_{2}(\{0\})\right\}$.

Proposition 3.4. Let $\mu$ a p-measure on $\mathbb{R}_{+}$such that $\mu(\{0\})<1$. Then $\psi_{\mu}$ is univalent in the left half-plane $i \mathbb{C}^{+}$, and $\psi_{\mu}\left(i \mathbb{C}^{+}\right)$is a region contained in the circle with diameter $(\mu(\{0\})-1,0)$; moreover $\psi_{\mu}\left(i \mathbb{C}^{+}\right) \cap \mathbb{R}_{+}=(\mu(\{0\})-1,0)$.

Let $\mu \in \mathcal{M}_{+}^{0}$. Denote by $\bar{\mu}$ the measure such that $\bar{\mu}(B)=\mu\left(B^{-1}\right)$ for any Borel set $B \subset \mathbb{R}_{+} \backslash\{0\}$. Denote $\mu^{s}:=\mu \otimes \bar{\mu}$.

Proposition 3.5. A p-measure $\mu \in \mathcal{M}_{+}^{0}$ satisfies $\bar{\mu}=\mu$ if and only if one of the following relations hold, for $z \neq 1$ and $|z|=1$,

1) $\Re \psi_{\mu}(z)=-1 / 2$;

2) $\left|R_{\mu}(z)\right|=1$;

3) $\left|\Sigma_{\mu}(z)\right|=1$, where $\Sigma_{\mu}$ is defined.

Proof. By the integral representation $(2.1)$ for $\psi_{\mu}(z)$, it is easy to verify that

$$
\psi_{\bar{\mu}}\left(\frac{1}{\bar{z}}\right)+\frac{1}{2}=-\left(\overline{\psi_{\mu}(z)}+\frac{1}{2}\right), \quad z \in \mathbb{C}^{+} .
$$

The first assertion of the lemma immediately follows from this formula and from the symmetry principle for analytic functions.

Recalling the definition of the function $R_{\mu}(z)$ and using (3.7), we get the relation

$$
R_{\bar{\mu}}\left(\frac{1}{\bar{z}}\right)=\frac{1}{\overline{R_{\mu}(z)}}, \quad z \in \mathbb{C}^{+} .
$$

As before the second assertion of the lemma immediately follows from (3.8) and from the symmetry principle for analytic functions.

Using (3.8), we obtain the formula

$$
\Sigma_{\bar{\mu}}\left(\frac{1}{\bar{z}}\right)=\frac{1}{\overline{\Sigma_{\mu}(z)}}
$$

for $z$ such that $\Sigma_{\mu}(z)$ is defined. We obtain the third assertion of the lemma as before.

We obtain, as an evident consequences of Proposition 3.5, that $\mu^{s}$ has the property $\bar{\mu}^{s}=\mu^{s}$. In addition, if $\mu_{1}$ and $\mu_{2}$ from the class $\mathcal{M}_{+}^{0}$ such that $\bar{\mu}_{1}=\mu_{1}$ and $\bar{\mu}_{2}=\mu_{2}$, then $\mu_{1} \otimes \mu_{2}$ has the same property as well. 
As for multiplicative free convolution we can formulate the limit problem for the multiplicative classical convolution $\circledast$. Let $\left\{\mu_{n k}: n \geq 1,1 \leq k \leq k_{n}\right\}, k_{n} \uparrow \infty$ as $n \rightarrow \infty$, be a triangular scheme of infinitesimal measures in $\mathcal{M}_{+}^{0}$. We shall

1) determine all $\mu \in \mathcal{M}_{+}^{0}$ such that $\mu^{(n)}=\delta_{1 / a_{n}} \circledast \mu_{n 1} \circledast \mu_{n 2} \cdots \circledast \mu_{n k_{n}} \rightarrow \mu$ in the weak topology, here $\left\{a_{n}\right\}$ is a sequence of positive numbers;

2 ) determine conditions under which $\mu^{(n)}$ converges weakly to a given $\rightarrow \mu$.

For measures $\mu_{n k} \in \mathcal{M}_{+}^{0}$ we denote by $\widehat{\mu}_{n k}$ the measures such that $\widehat{\mu}_{n k}((-\infty, x)):=$ $\mu_{n k}\left(\left(-\infty, a_{n k} x\right)\right), x>0$, where $a_{n k}:=\exp \left\{\int_{(1-\tau, 1+\tau)} \log u \mu_{n k}(d u)\right\}$. Here $\tau \in(0,1)$ is an arbitrary but fixed number.

A complete solution of this problem follows from the Limit Theorem for classical convolution $*$ (see [13], p. 310, [12]).

Theorem 3.6. Let $\mu_{n k}$ be a triangular scheme of infinitesimal probability measures in $\mathcal{M}_{+}^{0}$. Then we have

1. The family of limit measures of sequences $\mu^{(n)}=\delta_{1 / a_{n}} \circledast \mu_{n 1} \circledast \mu_{n 2} \circledast \cdots \circledast \mu_{n k_{n}}$ coincides with the family of $\circledast$-infinitely divisible measures.

2 . There exist positive constants $a_{n}$ such that the sequence $\mu^{(n)}=\delta_{1 / a_{n}} \circledast \mu_{n 1} \circledast \mu_{n 2} \circledast$ $\cdots \circledast \mu_{n k_{n}}$ converges weakly if, and only if, $\sigma_{n}$ converges weakly on $(0, \infty)$ to some finite nonnegative measure $\sigma^{\circledast}$ on $(0, \infty)$, where, for any Borel set $S \subset(0, \infty)$,

$$
\sigma_{n}(S):=\sum_{k=1}^{k_{n}} \int_{S} \frac{\log ^{2} u}{1+\log ^{2} u} \widehat{\mu}_{n k}(d u) .
$$

Then all admissible $a_{n}$ are of the form $a_{n}=\exp \left\{\alpha_{n}-\alpha+o(1)\right\}$, where $\alpha$ is an arbitrary finite number and

$$
\alpha_{n}=\sum_{k=1}^{k_{n}}\left(\int_{1-\tau}^{1+\tau} \log u \mu_{n k}(d u)+\int_{(0, \infty)} \frac{\log u}{1+\log ^{2} u} \widehat{\mu}_{n k}(d u)\right),
$$

and all possible limit measures $\mu \in \mathcal{M}_{+}^{0}$ have $m_{\mu}=\left\{\alpha, \sigma^{\circledast}\right\}$.

By $\mathcal{C}$ we denote $\mathrm{C}$. Carathéodory's class of analytic functions $F(z): \mathbb{D} \rightarrow\{z: \Re z \geq 0\}$. A function $F$ is in $\mathcal{C}$ if and only if it admits the following representation (Herglotz, G., Riesz, F.)

$$
F(z)=i a+\int_{[-\pi, \pi)} \frac{e^{i y}+z}{e^{i y}-z} \sigma(d y)
$$

where $a=\Im F(0)$ and $\sigma$ is finite nonnegative measure. The number $a$ and the measure $\sigma$ are uniquely determined by $F$.

Write the Stieltjes-Perron inversion formula for the function $F$ of the class $\mathcal{C}$ as follows:

$$
\kappa\left(y_{2}\right)-\kappa\left(y_{1}\right)=\frac{1}{\pi} \lim _{r \uparrow 1} \int_{y_{1}}^{y_{2}} \Re F\left(r e^{i \varphi}\right) d \varphi,
$$


where $-\pi \leq y_{1}<y_{2}<\pi$ are continuity points of the function $\kappa(y):=\sigma([-\pi, y)), y \in$ $[-\pi, \pi)$.

By $\mathcal{S}$ we denote J. Schur's class of analytic functions $\varphi(z): \mathbb{D} \rightarrow \overline{\mathbb{D}}$. The classes $\mathcal{C}$ and $\mathcal{S}$ are connected via

$$
\varphi(z)=\frac{1}{z} \frac{F(z)-F(0)}{F(z)+\overline{F(0)}}
$$

which induces a one-to-one correspondence between $\mathcal{C}$ and $\mathcal{S}$.

As it was proved in [10], (2.12) admits the following consequences.

Proposition 3.7. Let $\mu_{1}, \ldots, \mu_{n} \in \mathcal{M}_{*}$. There exist unique functions $Z_{1}(z), \ldots, Z_{n}(z)$ of the class $\mathcal{S}_{*}$ such that, for $z \in \mathbb{D}$,

$$
Z_{1}(z) \ldots Z_{n}(z)=z\left(Q_{\mu_{1}}\left(Z_{1}(z)\right)\right)^{n-1}, \quad \text { and } \quad Q_{\mu_{1}}\left(Z_{1}(z)\right)=\cdots=Q_{\mu_{n}}\left(Z_{n}(z)\right) .
$$

Moreover, $Q_{\mu_{1} \otimes \cdots \bowtie \mu_{n}}(z)=Q_{\mu_{1}}\left(Z_{1}(z)\right)$ for all $z \in \mathbb{D}$.

Let $\mu \in \mathcal{M}_{*}$. Denote by $\bar{\mu}$ the measure such that $\bar{\mu}(B)=\mu(-B)$ for any Borel set $B \subset \mathbb{T}$. Denote $\mu^{s}:=\mu \otimes \bar{\mu}$.

Proposition 3.8. A p-measure $\mu \in \mathcal{M}_{*}$ has the property $\bar{\mu}=\mu$ if and only if one of the following relations hold, for real $x$ such that $-1<x<1$,

1) $\Im \psi_{\mu}(x)=0$;

2) $\Im Q_{\mu}(x)=0$;

3) $\Im \Sigma_{\mu}(x)=0$, where $\Sigma_{\mu}$ is defined.

Proof. By the integral representation $(2.9)$ for $\psi_{\mu}(z)$, it is easy to verify that

$$
\psi_{\bar{\mu}}(\bar{z})=\overline{\psi_{\mu}(z)}, \quad z \in \mathbb{D} .
$$

The first assertion of the lemma immediately follows from this formula and from the symmetry principle for analytic functions.

Recalling the definition of the function $Q_{\mu}(z)$ (see (2.10)), we get the relation

$$
Q_{\bar{\mu}}(\bar{z})=\overline{Q_{\mu}(z)}, \quad z \in \mathbb{D} .
$$

As before the second assertion of the lemma immediately follows from (3.14) and from the symmetry principle for analytic functions.

Using (3.14), we obtain the formula

$$
\Sigma_{\bar{\mu}}(\bar{z})=\overline{\Sigma_{\mu}(z)}
$$

where $\Sigma_{\mu}(z)$ is defined. We obtain the third assertion of the lemma as before.

We need the following results for the convergence of $*$-infinitely divisible p-measures on $\mathbb{T}$ (see [14], [15]).

If $\nu$ is any finite measure on $\mathbb{T}$ the p-measure $e(\nu)$ associated with $\nu$ is defined as follows:

where $\nu^{n *}:=\nu * \cdots * \nu(n$ times $)$.

$$
e(\nu)=e^{-\nu(\mathbb{T})}\left(\delta_{0}+\nu+\frac{\nu^{2 *}}{2 !}+\cdots+\frac{\nu^{n *}}{n !}+\ldots\right)
$$


Theorem 3.9. Let $\left\{\mu_{n k}: n \geq 1, k=1, \ldots, k_{n}\right\}$, be a uniformly infinitesimal sequence of $p$-measures, and let $\mu_{n}=\mu_{n 1} * \cdots * \mu_{n k_{n}}$. Let $\beta_{n k}=e\left(\mu_{n k} * x_{n k}\right)$, where $x_{n k}$ is that element of the group $\mathbb{T}$ defined by the equation

$$
e^{i m x_{n k}}=\exp \left\{-i m \int_{[-\pi, \pi)} g(x) \mu_{n k}(d x)\right\}, \quad m \in \mathbb{Z} .
$$

Let $\lambda_{n}=\beta_{n 1} * \cdots * \beta_{n k_{n}} * x_{n}$, where $x_{n}=-\left(x_{n 1}+\cdots+x_{n k_{n}}\right)$. If one of the sequences $\left\{\lambda_{n}\right\}$ and $\left\{\mu_{n}\right\}$ is shift compact and no limit of its shifts has an idempotent factor, then

$$
\lim _{n \rightarrow \infty} \sup _{m \in K}\left|q_{\lambda_{n}}(m)-q_{\mu_{n}}(m)\right|=0
$$

for all compact set $K$ of $\mathbb{Z}$.

Theorem 3.10. Let $\left\{\mu_{n}\right\}$ be a sequence of $*$-infinitely divisible p-measures without idempotent divisors with representations $\left\{\left\langle a_{n}, \nu_{n}\right\rangle\right\}$ and $\mu$ is an infinitely divisible p-measure without idempotent divisors with a representation $\{\langle a, \nu\rangle\}$. The conditions $\nu_{n} \rightarrow \nu$ weakly and $a_{n} \rightarrow a$ as $n \rightarrow \infty$ are sufficient for the weak convergence $\mu_{n} \rightarrow \mu$.

This result is a simple consequence of Theorem 4.10 in [15].

The following proposition (see [14], p. 112) show that the representation (2.19) is not unique.

Proposition 3.11. There exist two finite nonnegative measures $\nu_{1} \neq \nu_{2}$ on $\mathbb{T}$ such that $\bar{\nu}_{1}=\nu_{2}$ and

$$
\exp \left\{\int_{[-\pi, \pi)}\left(e^{i m t}-1\right) \nu_{1}(d t)\right\}=\exp \left\{\int_{[-\pi, \pi)}\left(e^{i m t}-1\right) \nu_{2}(d t)\right\}, \quad m \in \mathbb{Z} .
$$

Proof. Following the arguments in [14], p. 112, we consider the function $f(x)=$ $4 \pi \sin (n x)$, where $x \in[-\pi, \pi)$ and $n \neq 0$ belongs to $\mathbb{Z}$. We see that

$$
\frac{1}{2 \pi} \int_{[-\pi, \pi)} e^{i m x} f(x) d x=q_{m}, \quad m \in \mathbb{Z}
$$

where $q_{m}=2 \pi i$ if $m=n, q_{m}=-2 \pi i$ if $m=-n$, and $q_{m}=0$ otherwise. Writing $f^{+}$and $f^{-}$for the positive and negative parts of $f$, we define two measures

$$
\nu_{1}(S)=\frac{1}{2 \pi} \int_{S} f^{+}(x) d x, \quad \nu_{2}(S)=\frac{1}{2 \pi} \int_{S} f^{-}(x) d x
$$

for all Borel sets on $[-\pi, \pi)$. Then $\nu_{1} \neq \nu_{2}$, but (3.17) holds which was to be proved. 


\section{Multiplicative free limit theorem in $\mathcal{M}_{+}$}

In Section 4 we prove Theorem 2.1. We denote here by $c$ positive absolute constants. For some measure $\nu$ and for some parameter $d$ we denote by $c(\nu), c(d)$, and $c(\nu, \tau)$ positive constants which depend on the measure $\nu$, on the parameter $d$, and on $\nu$ and $d$, respectively. By $c_{j}(\nu), c_{j}(d), c_{j}(\nu, d), j=1, \ldots$, we denote explicit positive constants depending on corresponding measures and parameters. In the first step we establish some properties of the measures $\left\{\mu_{n k}: n \geq 1,1 \leq k \leq k_{n}\right\}, k_{n} \uparrow \infty$, satisfying condition (2.8), and the corresponding transforms $\left\{R_{\mu_{n k}}(z): n \geq 1,1 \leq k \leq k_{n}\right\}$.

It is clear that the condition (2.8) is equivalent to the following one

$$
\max _{k=1, \ldots, k_{n}} \int_{\mathbb{R}_{+}} \frac{(u-1)^{2}}{1+u^{2}} \mu_{n k}(d u) \rightarrow 0, \quad n \rightarrow \infty
$$

Recall that $\widehat{\mu}_{n k}$ are the p-measures such that $\widehat{\mu}_{n k}((-\infty, x))=\mu_{n k}\left(\left(-\infty, a_{n k} x\right)\right)$, where $a_{n k}:=\int_{(1-d, 1+d)} u \mu_{n k}(d u)$. Here $d \in(0,1)$ is an arbitrary but fixed number. Since $\max _{k=1, \ldots, k_{n}}\left|a_{k n}-1\right| \rightarrow 0$ as $n \rightarrow \infty$, we conclude

$$
\varepsilon_{n}:=\max _{k=1, \ldots, k_{n}} \varepsilon_{n k} \rightarrow 0, \quad n \rightarrow \infty, \quad \text { where } \quad \varepsilon_{n k}:=\int_{\mathbb{R}_{+}} \frac{(u-1)^{2}}{1+u^{2}} \widehat{\mu}_{n k}(d u) .
$$

Let $\mu \in \mathcal{M}_{+}$with a finite second moment. Denote

$$
a(\mu):=\int_{\mathbb{R}_{+}} u \mu(d u), \quad \sigma^{2}(\mu):=\int_{\mathbb{R}_{+}} u^{2} \mu(d u)-\left(\int_{\mathbb{R}_{+}} u \mu(d u)\right)^{2} .
$$

Consider the p-measures $\rho_{n k}, k=1, \ldots, k_{n}$, such that, for all Borel set $B \subseteq \mathbb{R}_{+}$,

$$
\rho_{n k}(B)=\frac{1}{b_{n k}} \int_{B} \frac{\widehat{\mu}_{n k}(d u)}{1+u^{2}} \quad \text { with } \quad b_{n k}:=\int_{\mathbb{R}_{+}} \frac{\widehat{\mu}_{n k}(d u)}{1+u^{2}} .
$$

First let us prove that

$$
\left|a\left(\rho_{n k}\right)-1\right| \leq c(d) \varepsilon_{n k}, \quad n \geq n_{0}, k=1, \ldots, k_{n},
$$

where $n_{0}$ is a sufficiently large positive integer. Write

$$
b_{n k}\left(a\left(\rho_{n k}\right)-1\right)=I_{1}+I_{2}:=\left(\int_{\left|a_{n k} u-1\right| \leq d}+\int_{\left|a_{n k} u-1\right|>d}\right) \frac{u-1}{1+u^{2}} \widehat{\mu}_{n k}(d u) .
$$


We note that

$$
\begin{aligned}
I_{1} & =\frac{1}{2} \int_{\left|a_{n k} u-1\right| \leq d}(u-1) \widehat{\mu}_{n k}(d u)+\theta c(d) \int_{\left|a_{n k} u-1\right| \leq d}(u-1)^{2} \widehat{\mu}_{n k}(d u) \\
& =\frac{1}{2 a_{n k}} \int_{[1-d, 1+d]}\left(x-a_{n k}\right) \mu_{n k}(d x)+\theta c(d) \varepsilon_{n k}=\theta c(d) \varepsilon_{n k} .
\end{aligned}
$$

Here and in the sequel we denote by $\theta$ a real quantity such that $|\theta| \leq 1$. Furthermore,

$$
\left|I_{2}\right| \leq c(d) \int_{\left|a_{n k} u-1\right|>d} \frac{(u-1)^{2}}{1+u^{2}} \widehat{\mu}_{n k}(d u) \leq c(d) \varepsilon_{n k} .
$$

The last two estimates together with the obvious lower bound

$$
b_{n k} \geq \frac{1}{4}, \quad n \geq n_{0}, k=1, \ldots, k_{n},
$$

prove (4.3).

Secondly we shall prove that

$$
\frac{1}{2} \varepsilon_{n k} \leq \sigma^{2}\left(\rho_{n k}\right) \leq c(d) \varepsilon_{n k}, \quad n \geq n_{0}, k=1, \ldots, k_{n} .
$$

Indeed, using (4.3) and (4.4), we have the relation, for $n \geq n_{0}, k=1, \ldots, k_{n}$,

$$
\begin{aligned}
\sigma^{2}\left(\rho_{n k}\right) & =\frac{1}{b_{n k}} \int_{\mathbb{R}_{+}} \frac{\left(u-a\left(\rho_{n k}\right)\right)^{2}}{1+u^{2}} \widehat{\mu}_{n k}(d u) \leq \frac{2}{b_{n k}} \int_{\mathbb{R}_{+}} \frac{(u-1)^{2}}{1+u^{2}} \widehat{\mu}_{n k}(d u) \\
& +\frac{2\left(a\left(\rho_{n k}\right)-1\right)^{2}}{b_{n k}} \int_{\mathbb{R}_{+}} \frac{\widehat{\mu}_{n k}(d u)}{1+u^{2}} \leq \frac{(2+c(d)) \varepsilon_{n k}}{b_{n k}} \leq c(d) \varepsilon_{n k} .
\end{aligned}
$$

In view of (4.3) and (4.4), we obtain the lower bound

$$
\begin{aligned}
\sigma^{2}\left(\rho_{n k}\right) & \geq \frac{1}{b_{n k}} \int_{\mathbb{R}_{+}} \frac{(u-1)^{2}}{1+u^{2}} \widehat{\mu}_{n k}(d u)-2\left|a\left(\rho_{n k}\right)-1\right|\left(\frac{1}{b_{n k}} \int_{\mathbb{R}_{+}} \frac{(u-1)^{2}}{1+u^{2}} \widehat{\mu}_{n k}(d u)\right)^{1 / 2} \\
& \geq\left(\frac{1}{b_{n k}}-4 c(d) \sqrt{\varepsilon_{n k}}\right) \varepsilon_{n k} \geq \frac{1}{2} \varepsilon_{n k}, \quad n \geq n_{0}, \quad k=1, \ldots k_{n} .
\end{aligned}
$$

The estimate (4.5) follows from the last two bounds.

For every $k=1, \ldots, k_{n}$ we have, taking into account (3.4),

$$
R_{\widehat{\mu}_{n k}}(z)=c_{n k} z+z \int_{(0, \infty)} \frac{\tau_{n k}(d u)}{u-z}, \quad z \in \mathbb{C} \backslash[0, \infty), k=1, \ldots, k_{n},
$$


where $c_{n k}$ are nonnegative constants and $\tau_{n k}$ are nonnegative measures such that

$$
\int_{(0, \infty)} \frac{\tau_{n k}(d u)}{1+u}<\infty
$$

Since

$$
\Im R_{\widehat{\mu}_{n k}}(i)=\Im \frac{\psi_{\widehat{\mu}_{n k}}(i)}{1+\psi_{\widehat{\mu}_{n k}}(i)}=a\left(\rho_{n k}\right)\left(1+\frac{\sigma^{2}\left(\rho_{n k}\right)}{1+a^{2}\left(\rho_{n k}\right)}\right),
$$

and

$$
\Re R_{\widehat{\mu}_{n k}}(i)=\Re \frac{\psi_{\widehat{\mu}_{n k}}(i)}{1+\psi_{\widehat{\mu}_{n k}}(i)}=-\frac{\sigma^{2}\left(\rho_{n k}\right)}{1+a^{2}\left(\rho_{n k}\right)},
$$

we have, by (4.3) and (4.5), for $n \geq n_{0}, k=1, \ldots, k_{n}$,

$$
\frac{1}{8} \varepsilon_{n k} \leq \int_{(0, \infty)} \frac{\tau_{n k}(d u)}{1+u^{2}} \leq c(d) \varepsilon_{n k}, \quad \text { and } \quad\left|R_{\widehat{\mu}_{n k}}(i)-i\right| \leq c(d) \varepsilon_{n k} .
$$

In view of the bound

$$
\left|\frac{1}{u-z}\right| \leq \frac{Q(z)}{\sqrt{1+u^{2}}} \quad z \in \mathbb{C}^{+}, u>0
$$

where

$$
Q(z):=1+\frac{1}{|z|} \text { if } z \in \mathbb{C}^{+} \cap\left(i \mathbb{C}^{+}\right) \text {and } Q(z):=2\left(1+\frac{1+\Re z}{\Im z}\right) \text { if } z \in \mathbb{C}^{+} \cap\left(-i \mathbb{C}^{+}\right),
$$

we obtain from (4.10) and (4.11)

$$
\begin{aligned}
\left|\frac{R_{\widehat{\mu}_{n k}}(z)}{z}-1\right| & \leq\left|\frac{R_{\widehat{\mu}_{n k}}(z)}{z}-\frac{R_{\widehat{\mu}_{n k}}(i)}{i}\right|+\left|R_{\widehat{\mu}_{n k}}(i)-i\right| \\
& \leq|z-i| \int_{\mathbb{R}_{+}} \frac{\tau_{n k}(d u)}{\sqrt{1+u^{2}}|u-z|}+c(d) \varepsilon_{n k} \leq c(d) \varepsilon_{n k}(|z|+1) Q(z)
\end{aligned}
$$

for $z \in \mathbb{C}^{+}$, and

$$
\begin{aligned}
\mid \frac{R_{\widehat{\mu}_{n k}}\left(z_{1}\right)}{z_{1}} & -\frac{R_{\widehat{\mu}_{n k}}\left(z_{2}\right)}{z_{2}} \mid \leq \int_{\mathbb{R}_{+}} \frac{\left|z_{1}-z_{2}\right| \tau_{n k}(d u)}{\left|u-z_{1}\right|\left|u-z_{2}\right|} \\
& \leq\left|z_{1}-z_{2}\right| Q\left(z_{1}\right) Q\left(z_{2}\right) \int_{\mathbb{R}_{+}} \frac{\tau_{n k}(d u)}{u^{2}+1} \leq c(d) \varepsilon_{n k}\left|z_{1}-z_{2}\right| Q\left(z_{1}\right) Q\left(z_{2}\right)
\end{aligned}
$$

for $z_{1}, z_{2} \in \mathbb{C}^{+}$.

In addition, using (4.10) and (4.12), we easily conclude that

$$
c_{1}(d) \varepsilon_{n k} \Im z \leq \Im\left(R_{\widehat{\mu}_{n k}}(z) / z\right) \mid \leq c_{2}(d) \varepsilon_{n k} \Im z \quad \text { and } \quad\left|\Re\left(R_{\widehat{\mu}_{n k}}(z) / z\right)-1\right| \leq c(d) \varepsilon_{n k}
$$


for $z \in \mathbb{C}$ such that $1 / 4 \leq|z| \leq 2$ and $\pi / 4 \leq \arg z \leq \pi$, and for $n \geq n_{0}, k=1, \ldots, k_{n}$. Hence for the same $z$ and $n, k$ the following relations hold

$$
\left|R_{\widehat{\mu}_{n k}}(z) / z-1\right| \leq c(d) \varepsilon_{n k} \quad \text { and } \quad c_{3}(d) \leq \frac{\arg \left(R_{\widehat{\mu}_{n k}}(z) / z\right)}{\varepsilon_{n k}(\pi-\arg z)} \leq c_{4}(d) .
$$

The relations (4.14) imply that the inverse functions $R_{\widehat{\mu}_{n k}}^{(-1)}(z)$ exist and are analytic in the domain $D_{1}:=\{z \in \mathbb{C}: 1 / 2<|z|<3 / 2,3 \pi / 8<\arg z<\pi\}$. Moreover, for $z \in D_{1}$ and for $n \geq n_{0}, k=1, \ldots, k_{n}$,

$$
\left|\left(R_{\widehat{\mu}_{n k}}^{(-1)}(z) / z\right)-1\right| \leq c(d) \varepsilon_{n k} \quad \text { and } \quad c_{5}(d) \leq-\frac{\arg \left(R_{\widehat{\mu}_{n k}}^{(-1)}(z) / z\right)}{\varepsilon_{n k}(\pi-\arg z)} \leq c_{6}(d) .
$$

Proof of Theorem 2.1. Sufficiency.

By Proposition 3.1, there exist unique functions $Z_{n 1}(z), \ldots, Z_{n k_{n}}(z)$ of class $\mathcal{K}$ such that, for all $z \in \mathbb{C}^{+}$,

$$
\frac{R_{\widehat{\mu}_{n 1}}\left(Z_{n 1}(z)\right)}{z}=\frac{R_{\widehat{\mu}_{n 1}}\left(Z_{n 2}(z)\right)}{Z_{n 1}(z)} \ldots \frac{R_{\widehat{\mu}_{n n}}\left(Z_{n k_{n}}(z)\right)}{Z_{n k_{n}}(z)}
$$

and

$$
R_{\widehat{\mu}_{n 1}}\left(Z_{n 1}(z)\right)=\cdots=R_{\widehat{\mu}_{n n}}\left(Z_{n k_{n}}(z)\right) \text {. }
$$

Moreover, $R_{n}(z):=R_{\widehat{\mu}_{n}}(z)=R_{\widehat{\mu}_{n k}}\left(Z_{n k}(z)\right), k=1, \ldots, k_{n}$, where $\widehat{\mu}_{n}:=\widehat{\mu}_{n 1} \otimes \cdots \otimes \widehat{\mu}_{n k_{n}}$.

By Propositions 3.3, 3.4, we see that the functions $\Sigma_{\widehat{\mu}_{n}}(z), \Sigma_{\widehat{\mu}_{n k}}(z), k=1, \ldots, k_{n}$, are defined on some domain $\Omega \subseteq i \mathbb{C}^{+}$which contains the interval $\left(1-1 / \Delta_{n}, 0\right)$, where the parameter $\Delta_{n}:=\max _{k=1, \ldots, k_{n}} \mu_{n k}(\{0\})$ satisfies $\Delta_{n} \leq \max _{k=1, \ldots, k_{n}} \varepsilon_{n k}$ and tends to 0 as $n \rightarrow \infty$. By (4.16) and (4.17), we have

$$
\Sigma_{\widehat{\mu}_{n}}(z)=\Sigma_{\widehat{\mu}_{n 1}}(z) \ldots \Sigma_{\widehat{\mu}_{n k_{n}}}(z), \quad z \in \Omega
$$

Denote $\eta_{n}:=\sum_{k=1}^{k_{n}} \varepsilon_{n k}$. Recall that, by the assumption of the theorem, $\eta_{n} \rightarrow \sigma([0, \infty])$ as $n \rightarrow \infty$. Hence $\eta_{n} \leq \sigma([0, \infty])+1$ for $n \geq n_{0}$. We see that $\Sigma_{\widehat{\mu}_{n}}(z), n \geq n_{0}$, admit an analytic continuation in $D_{1}$ and there, by (4.15),

$$
\exp \left\{-c(d) \eta_{n}\right\} \leq\left|\Sigma_{\widehat{\mu}_{n}}(z)\right|=\left|\Sigma_{\widehat{\mu}_{n 1}}(z)\right| \ldots\left|\Sigma_{\widehat{\mu}_{n k_{n}}}(z)\right| \leq \exp \left\{c(d) \eta_{n}\right\}
$$

and

$$
c_{5}(d) \eta_{n} \leq-\frac{\arg \Sigma_{\widehat{\mu}_{n}}(z)}{\pi-\arg z}=-\frac{\arg \Sigma_{\widehat{\mu}_{n 1}}(z)+\cdots+\arg \Sigma_{\widehat{\mu}_{n k_{n}}}(z)}{\pi-\arg z} \leq c_{6}(d) \eta_{n} .
$$

In addition we note that, by (4.19) and (4.20), for $z \in D_{2}:=\{z \in \mathbb{C}: 1 / 2<|z|<$ $\left.3 / 2, \pi-c_{7}(d)<\arg z<\pi\right\}$ with sufficiently small $c_{7}(d)$ and $n \geq n_{0}, k=1, \ldots, k_{n}$,

$$
\Sigma_{\widehat{\mu}_{n}}(z)=\frac{\left(R_{\widehat{\mu}_{n k}}\left(Z_{n k}\right)^{(-1)}(z)\right.}{z}=\frac{Z_{n k}^{(-1)}\left(R_{\widehat{\mu}_{n k}}^{(-1)}(z)\right)}{z},
$$


and hence, for the same $z, n, k$,

$$
\frac{Z_{n k}\left(z \Sigma_{\widehat{\mu}_{n}}(z)\right)}{z \Sigma_{\widehat{\mu}_{n}}(z)}=\frac{R_{\widehat{\mu}_{n k}}^{(-1)}(z)}{z} \frac{1}{\Sigma_{\widehat{\mu}_{n}}(z)} .
$$

By (4.15), (4.19) and (4.20), we see from (4.21) that there exist points $z_{n k}$ such that $z_{n k} \in\left\{z \in \mathbb{C}: e^{-c_{8}(d) \eta_{n}} \leq|z| \leq e^{c_{8}(d) \eta_{n}}, \pi-c_{7}(d)<\arg z<\pi-c_{7}(d) / 2\right\}$, where

$$
e^{-c(d) \eta_{n}} \leq\left|Z_{n k}\left(z_{n k}\right) / z_{n k}\right| \leq e^{c(d) \eta_{n}} \quad \text { and } \quad \frac{1}{c(d)} \leq \frac{\arg \left(Z_{n k}\left(z_{n k}\right) / z_{n k}\right)}{\eta_{n}\left(\pi-\arg z_{n k}\right)} \leq c(d)
$$

for $n \geq n_{0}, k=1, \ldots, k_{n}$ with some positive constant $c(d)>1$.

The functions $Z_{n k}, n \geq 1, k=1, \ldots, k_{n}$, are in $\mathcal{K}$ and therefore, by (3.4),

$$
Z_{n k}(z)=d_{n k} z+z \int_{(0, \infty)} \frac{\nu_{n k}(d u)}{u-z} \quad z \in \mathbb{C}^{+}
$$

where $d_{n k}$ are nonnegative constants and $\nu_{n k}$ are nonnegative measures such that

$$
\int_{(0, \infty)} \frac{\nu_{n k}(u)}{1+u}<\infty
$$

Applying the bounds (4.22) to the integral representation (4.23), we easily obtain the following estimates, for all $n \geq n_{0}, k=1, \ldots, k_{n}$,

$$
e^{-c_{11}(d) \eta_{n}} \leq d_{n k}+\int_{(0, \infty} \frac{\left(u+c_{9}(d)\right) \nu_{n k}(d u)}{\left(u+c_{9}(d)\right)^{2}+c_{10}(d)} \leq e^{c_{11}(d) \eta_{n}}
$$

and

$$
c_{12}(d) \eta_{n} \leq \int_{(0, \infty)} \frac{\nu_{n k}(u)}{1+u^{2}} \leq c_{13}(d) \eta_{n}
$$

Then we obtain, using (4.11) and (4.24),

$$
\left|Z_{n k}(z)\right| \leq c(\sigma, d)|z| Q(z), \quad z \in \mathbb{C}^{+}, n \geq n_{0}, k=1, \ldots, k_{n} .
$$

On the other hand, by (4.24), (4.25) and the lower bound

$$
\frac{1}{|u-z|^{2}} \geq \frac{1}{(u+|\Re z|)^{2}+(\Im z)^{2}} \geq \frac{1}{2} \frac{1}{u^{2}+|z|^{2}} \geq \frac{1}{2} \frac{1}{1+|z|^{2}} \cdot \frac{1}{1+u^{2}}, u>0, z \in \mathbb{C},
$$

we have, for the same $z, n, k$,

$$
\left|Z_{n k}(z)\right| \geq|z| \Im\left(Z_{n k}(z) / z\right)=|z| \Im z \int_{(0, \infty)} \frac{\nu_{n k}(d u)}{(u-\Re z)^{2}+(\Im z)^{2}} \geq \frac{c_{12}(d)}{2} \eta_{n} \frac{|z| \Im z}{1+|z|^{2}}
$$


and

$$
\begin{aligned}
\left|Z_{n k}(z)\right| & \geq|z| \mid \Re\left(Z_{n k}(z) / z|=| z|| d_{n k}+\int_{(0, \infty)} \frac{(u-\Re z) \nu_{n k}(d u)}{(u-\Re z)^{2}+(\Im z)^{2}} \mid\right. \\
& \geq \max \left\{0, \frac{1}{2\left(1+|z|^{2}\right)}\left(d_{n k}+\int_{(0, \infty)} \frac{u \nu_{n k}(d u)}{1+u^{2}}\right)\right. \\
& \left.-(1+\operatorname{sign}(\Re z)) \Re z \int \frac{\nu_{n k}(d u)}{(u-\Re z)^{2}+(\Im z)^{2}}\right\} \geq c(d)|z| g_{n}(z),
\end{aligned}
$$

where

$$
g_{n}(z):=\max \left\{0,\left(e^{-c_{11}(d) \eta_{n}}-c(d) \eta_{n}\right) \frac{1}{2\left(1+|z|^{2}\right)}-(1+\operatorname{sign}(\Re z)) c_{14}(d) \eta_{n} \Re z \frac{1+(\Re z)^{2}}{(\Im z)^{2}}\right\} .
$$

Note that the estimate (4.28) is only useful if $\eta_{n}$ is sufficiently small.

Finally we get from (4.27) and (4.28), for $z \in \mathbb{C}^{+}, n \geq n_{0}, k=1, \ldots, k_{n}$.

$$
\left|Z_{n k}(z)\right| \geq \rho_{n}(z):=|z|\left(\frac{c_{12}(d)}{2} \eta_{n} \frac{|z| \Im z}{1+|z|^{2}}+c(d) g_{n}(z)\right) .
$$

Using (4.12), we deduce

$$
\left|\frac{R_{\widehat{\mu}_{n k}}\left(Z_{n k}(z)\right)}{Z_{n k}(z)}-1\right| \leq c(\sigma, d) \varepsilon_{n k}\left(\left|Z_{n k}(z)\right|+1\right) Q\left(Z_{n k}(z)\right)
$$

for $z \in \mathbb{C}^{+}, n \geq n_{0}$, and $k=1, \ldots, k_{n}$.

If $\Re Z_{n k}(z) \geq 0$, then, using $\sin (x) \geq \frac{2}{\pi} x$ for $0 \leq x \leq \pi / 2$, we obtain

$$
\Im Z_{n k}(z)=\left|Z_{n k}(z)\right| \sin \left(\arg Z_{n k}(z)\right) \geq \frac{2}{\pi}\left|Z_{n k}(z)\right| \arg Z_{n k}(z) \geq \frac{2}{\pi}\left|Z_{n k}(z)\right| \arg z
$$

and we get, by (4.26), (4.29), and (4.31),

$$
Q\left(Z_{n k}(z)\right) \leq 2+2 \frac{1+\left|Z_{n k}(z)\right|}{\Im Z_{n k}} \leq 2+c(\sigma, d) \frac{Q(z)(1+|z|)^{2}}{\rho_{n}(z) \Im z} .
$$

If $\Re Z_{n k}(z) \leq 0$, we have, by (4.29),

$$
Q\left(Z_{n k}(z)\right) \leq 1+\frac{1}{\left|Z_{n k}(z)\right|} \leq 1+\frac{1}{\rho_{n}(z)} .
$$

Applying (4.26), (4.32), and (4.33) to (4.30), we finally deduce

$$
\left|\frac{R_{\widehat{\mu}_{n k}}\left(Z_{n k}(z)\right)}{Z_{n k}(z)}-1\right| \leq c(\sigma, d) \varepsilon_{n k} Q_{1}(z):=c(\sigma, d) \varepsilon_{n k} \frac{Q^{2}(z)(1+|z|)^{3}}{\rho_{n}(z) \Im z}
$$

for $z \in \mathbb{C}^{+}, n \geq n_{0}$, and $k=1, \ldots, k_{n}$.

Introduce the domain $D(T):=\{z \in \mathbb{C}: 1 / T<\Im z<T,-T<\Re z<T\}$ for sufficiently large $T>c(\sigma, d)>1$. 
We shall estimate below the function $\rho_{n}(z)$ for $z \in D(T)$. First we assume that $\eta_{n}>\delta_{0} T^{-7}$ with sufficiently small $\delta_{0}=\delta_{0}(\sigma, d)$. In this case we obtain from (4.29) that $\rho_{n}(z) \geq c(\sigma, d) T^{-10}$. Now we consider the case where $\eta_{n} \leq \delta_{0} T^{-7}$. Then, it is easy to see, that the function $g_{n}(z)$ admits the estimate

$$
g_{n}(z) \geq c(\sigma, d) T^{-2}\left(1-10 e^{c_{11}(d) \eta_{n}} c_{14}(d) \eta_{n} T^{7}\right) \geq \frac{1}{2} c(\sigma, d) T^{-2}
$$

and we obtain that $\rho_{n}(z) \geq \frac{1}{2} c(\sigma, d) T^{-3}$. Hence we finally have

$$
\rho_{n}(z) \geq c(\sigma, d) T^{-10}, \quad z \in D(T) .
$$

Using this bound we see that the following estimate holds

$$
Q_{1}(z) \leq c(\sigma, d) T^{18}, \quad z \in D(T) .
$$

It follows from (4.34) and (4.36) that the right-hand side of (4.34) does not exceed $1 / 2$ for $z \in D\left(\delta \varepsilon_{n}^{-1 / 18}\right)$ with sufficiently small $\delta=\delta(\sigma, d)>0$. Using series expansion of the function $\log (1-z)$ for $|z|<1$ and (4.34), we easily obtain, for $n \geq n_{0}, k=1, \ldots, k_{n}$,

$$
\log \frac{R_{\widehat{\mu}_{n k}}\left(Z_{n k}(z)\right)}{Z_{n k}(z)}=\frac{R_{\widehat{\mu}_{n k}}\left(Z_{n k}(z)\right)}{Z_{n k}(z)}-1+r_{n k}(z), \quad z \in D\left(\delta \varepsilon_{n}^{-1 / 18}\right),
$$

where the function $r_{n k}(z)$ is analytic in $D\left(\delta \varepsilon_{n}^{-1 / 18}\right)$ and admits the following estimate

$$
\left|r_{n k}(z)\right| \leq c(\sigma, d) \varepsilon_{n k}^{2} Q_{1}^{2}(z) .
$$

In (4.37) we choose the principal branch of the logarithm.

Let us return to the relation (4.17). In view of (4.26) and (4.34), we have, for $z \in$ $D\left(\delta \varepsilon_{n}^{-1 / 18}\right), n \geq n_{0}$ and $k=2, \ldots, k_{n}$,

$$
\begin{aligned}
\left|Z_{n 1}(z)-Z_{n k}(z)\right| & \leq\left|R_{\widehat{\mu}_{n 1}}\left(Z_{n 1}(z)\right)-Z_{n 1}(z)\right|+\left|R_{\widehat{\mu}_{n k}}\left(Z_{n k}(z)\right)-Z_{n k}(z)\right| \\
& \leq c(\sigma, d) \varepsilon_{n}|z| Q(z) Q_{1}(z) .
\end{aligned}
$$

Denote

$$
q_{n k}(z):=\frac{R_{\widehat{\mu}_{n k}}\left(Z_{n k}(z)\right)}{Z_{n k}(z)}-\frac{R_{\widehat{\mu}_{n k}}\left(Z_{n 1}(z)\right)}{Z_{n 1}(z)}, \quad z \in \mathbb{C}^{+}, n \geq n_{0}, k=2, \ldots, k_{n} .
$$

By (4.13), it follows that

$$
\left|q_{n k}(z)\right| \leq c(d) \varepsilon_{n k}\left|Z_{n k}(z)-Z_{n 1}(z)\right| Q\left(Z_{n k}(z)\right) Q\left(Z_{n 1}(z)\right)
$$

for $z \in D\left(\delta \varepsilon_{n}^{-1 / 18}\right), n \geq n_{0}$ and $k=1, \ldots, k_{n}$. Taking into account (4.39) and (4.32), (4.33), (4.35), and (4.36) we have, for $z \in D(T)$ with $T \leq \delta \varepsilon_{n}^{-1 / 18}$ and $n \geq n_{0}, k=$ $1, \ldots, k_{n}$

$$
\left|q_{n k}(z)\right| \leq c(\sigma, d) \varepsilon_{n k} \varepsilon_{n} T^{51}
$$


Consider the functions

$$
\begin{aligned}
f_{n k}(z) & :=(1-z)\left(1+\psi_{\widehat{\mu}_{n k}}(z)\right)-1=z \int_{\mathbb{R}_{+}} \frac{(u-1) \widehat{\mu}_{n k}(d u)}{1-u z} \\
& =\frac{z}{1-z}\left(\int_{\mathbb{R}_{+}} \frac{u^{2}-1}{u^{2}+1} \widehat{\mu}_{n k}(d u)+\int_{\mathbb{R}_{+}} \frac{u+z}{1-u z} \frac{(u-1)^{2}}{u^{2}+1} \widehat{\mu}_{n k}(d u)\right)
\end{aligned}
$$

for $z \in \mathbb{C}^{+}, n=1, \ldots, k=1, \ldots, k_{n}$. It is easy to see that

$$
\frac{R_{\mu_{n k}}(z)}{z}-1=\frac{1-z}{z} f_{n k}(z)+\theta_{n k}(z):=\frac{1-z}{z}\left(f_{n k}(z)-\frac{f_{n k}^{2}(z)}{1+f_{n k}(z)}\right) .
$$

Since by (4.11), for $u>0$ and $z \in \mathbb{C}^{+}$,

$$
\frac{u}{|1-u z|} \leq \frac{u Q(z)}{\sqrt{1+u^{2}}} \text { and } \quad \frac{1}{|1-u z|} \leq \frac{Q(z)}{\sqrt{1+u^{2}}}
$$

we obtain from (4.42), for $z \in \mathbb{C}^{+}$and $n \geq n_{0}, k=1, \ldots, k_{n}$,

$$
\left|\int_{\mathbb{R}_{+}} \frac{u+z}{1-u z} \frac{(u-1)^{2}}{u^{2}+1} \widehat{\mu}_{n k}(d u)\right| \leq(1+|z|) Q(z) \int_{\mathbb{R}_{+}} \frac{(u-1)^{2}}{u^{2}+1} \widehat{\mu}_{n k}(d u) \leq \varepsilon_{n k}(1+|z|) Q(z) .
$$

Moreover, recalling the definition of $\widehat{\mu}_{n k}$, it is not difficult to deduce the following bound, for $n \geq n_{0}, k=1, \ldots, k_{n}$,

$$
\begin{aligned}
& \mid\left|\int_{\mathbb{R}_{+}} \frac{u^{2}-1}{u^{2}+1} \widehat{\mu}_{n k}(d u)\right| \leq\left|\int_{\left\{u \geq 0,\left|a_{n k} u-1\right|<d\right\}}(u-1) \widehat{\mu}_{n k}(d u)\right| \\
&+c(d) \int_{\left\{u \geq 0,\left|a_{n k} u-1\right|<d\right\}}(u-1)^{2} \widehat{\mu}_{n k}(d u)+\int_{\left\{u \geq 0,\left|a_{n k} u-1\right| \geq d\right\}} \widehat{\mu}_{n k}(d u) \leq c(d) \varepsilon_{n k} .
\end{aligned}
$$

The estimates (4.44) and (4.45) together imply

$$
\left|f_{n k}(z)\right| \leq c(d) \varepsilon_{n k} \frac{|z|(1+|z|)}{|1-z|} Q(z)
$$

for $z \in \mathbb{C}^{+}, n \geq n_{0}$ and $k=1, \ldots, k_{n}$. Since, as it is easy to see, $\left|f_{n k}(z)\right| \leq 1 / 2$ for $z \in D\left(\delta \varepsilon_{n}^{-1 / 18}\right)$ and hence $\left|1+f_{n k}(z)\right| \geq 1 / 2$ for the same $z, n, k$, it follows immediately from (4.46) that

$$
\left|\theta_{n k}(z)\right| \leq c(d) \varepsilon_{n k}^{2} \frac{|z|(1+|z|)^{2}}{|1-z|} Q^{2}(z)
$$

for $z \in D\left(\delta \varepsilon_{n}^{-1 / 18}\right)$ and $n \geq n_{0}, k=1, \ldots, k_{n}$. Denote

$$
r_{n}(z)=\sum_{k=1}^{k_{n}} r_{n k}(z), \quad q_{n}(z)=\sum_{k=1}^{k_{n}} q_{n k}(z), \quad \theta_{n}(z)=\sum_{k=1}^{k_{n}} \theta_{n k}\left(Z_{n 1}(z)\right) .
$$


Using (4.16), (4.37), (4.40), and (4.43), we have, for $n \geq n_{0}$,

$$
\begin{aligned}
\frac{R_{\widehat{\mu}_{n 1}}\left(Z_{n 1}(z)\right)}{z} & =\exp \left\{\frac{1-Z_{n 1}(z)}{Z_{n 1}(z)} \sum_{k=1}^{k_{n}} f_{n k}\left(Z_{n 1}(z)\right)\right. \\
& \left.\quad+\sum_{k=1}^{k_{n}} r_{n k}(z)+\sum_{k=1}^{k_{n}} q_{n k}(z)+\sum_{k=1}^{k_{n}} \theta_{n k}\left(Z_{n 1}(z)\right)\right\} \\
& =\exp \left\{\frac{1-Z_{n 1}(z)}{Z_{n 1}(z)} \sum_{k=1}^{k_{n}} f_{n k}\left(Z_{n 1}(z)\right)+r_{n}(z)+q_{n}(z)+\theta_{n}(z)\right\} \\
& =\exp \left\{\int \frac{u+1}{u-1} \sigma_{n}(d u)+\int_{\mathbb{R}_{+}} \frac{u+Z_{n 1}(z)}{1-u Z_{n 1}(z)} \sigma_{n}(d u)+r_{n}(z)+q_{n}(z)+\theta_{n}(z)\right\} .
\end{aligned}
$$

By (4.38), (4.41) and (4.36), we obtain, for $z \in D(T)$ with $T \leq \delta \varepsilon_{n}^{-1 / 18}$ and $n \geq n_{0}$,

$$
\left|r_{n}(z)\right|+\left|q_{n}(z)\right| \leq c(\sigma, d) \varepsilon_{n} \sum_{k=1}^{k_{n}} \varepsilon_{n k}\left(Q_{1}^{2}(z)+T^{51}\right) \leq c(\sigma, d) \varepsilon_{n} T^{51} .
$$

In addition, by (4.26), (4.31)-(4.33), (4.35), and (4.47) we conclude, for the same $z$ and $n$ as above,

$$
\left|\theta_{n}(z)\right| \leq c(d) \varepsilon_{n} \sum_{k=1}^{k_{n}} \varepsilon_{n k} \frac{\left|Z_{n 1}(z)\right|\left(1+\left|Z_{n 1}(z)\right|\right)^{2}}{\left|1-Z_{n 1}(z)\right|} Q^{2}\left(Z_{n 1}(z)\right) \leq c(\sigma, d) \varepsilon_{n} T^{39} .
$$

From (4.49) and (4.50) we see that, for sufficiently large $n \geq n_{0}$,

$$
\left|r_{n}(z)\right|+\left|q_{n}(z)\right|+\left|\theta_{n}(z)\right| \leq c(\sigma, d) \varepsilon_{n}^{1 / 52}, \quad z \in D\left(\varepsilon_{n}^{-1 / 52}\right) .
$$

Denote

$$
a_{n}^{\prime}:=\exp \left\{\alpha+o(1)-\int_{\mathbb{R}_{+}} \frac{u+1}{u-1} \sigma_{n}(d u)\right\}
$$

By (4.45),

$$
\left|\log a_{n}^{\prime}\right| \leq c_{15}(d)+|\alpha|<\infty \text { for all } n \geq n_{0},
$$

and we can rewrite (4.48) in the form

$$
\frac{R_{\widehat{\mu}_{n 1}}\left(Z_{n}(z)\right)}{z}=\exp \left\{\alpha+o(1)+\int_{\mathbb{R}_{+}} \frac{u+Z_{n}(z)}{1-u Z_{n}(z)} \sigma_{n}(d u)+\Delta_{n}(z)\right\},
$$

where $Z_{n}(z):=Z_{n 1}\left(a_{n}^{\prime} z\right)$, and $\Delta_{n}(z)$ is an analytic function in $D\left(e^{-c_{15}(d)-\alpha} \varepsilon_{n}^{-1 / 52}\right)$, where it admits the following estimate

$$
\left|\Delta_{n}(z)\right| \leq c(\sigma, d) \varepsilon_{n}^{1 / 52} .
$$


Return to the representation (4.23) for the functions $Z_{n 1}(z)$. By (4.24), (4.52), and the vague compactness theorem (see [13], p. 179), we conclude that there exist a subsequence $\left\{n^{\prime}\right\}$ such that

$$
d_{n^{\prime} 1} \rightarrow d_{1} \quad a_{n^{\prime}} \rightarrow a, \quad \text { and } \quad \int_{(0, \infty} \frac{\nu_{n^{\prime} 1}(d u)}{1+u} \rightarrow d_{2}, \quad n^{\prime} \rightarrow \infty,
$$

where $d_{j} \geq 0, j=1,2, a>0$, and $\left\{\nu_{n^{\prime} 1}(d u) /(1+u)\right\}$ converges in the vague topology to some nonnegative measure $\nu_{1}$ such that $\nu_{1}\left(\mathbb{R}_{+}\right) \leq d_{2}$. Rewrite the formula (4.23) with $k=1$ in the form

$$
Z_{n^{\prime} 1}(z)=d_{n^{\prime} 1} z+z \int_{(0, \infty}\left(\frac{u+1}{u-z}-1\right) \frac{\nu_{n^{\prime} 1}(d u)}{1+u}+z \int_{(0, \infty} \frac{\nu_{n^{\prime} 1}(d u)}{1+u} .
$$

Since the kernel under the integral sign in the last formula tends to 0 as $u \rightarrow \infty$ uniformly in $z$ from every compact set in $\mathbb{C}^{+}$, we obtain, by the Helly-Bray lemma (see [13], p. 181),

$$
Z_{n^{\prime} 1}(z) \rightarrow d_{1} z+z \int_{\mathbb{R}_{+}} \frac{u+1}{u-z} \nu_{1}(d u)+\left(d_{2}-\nu_{1}\left(\mathbb{R}_{+}\right)\right) z
$$

uniformly on every compact set in $\mathbb{C}^{+}$. Finally we obtain from this relation that $Z_{n^{\prime}}(z) \rightarrow$ $Z(z)$ as $n^{\prime} \rightarrow \infty$ uniformly on every compact set in $\mathbb{C}^{+}$, where $Z(z) \in \mathcal{N}$ and $Z(z) / z \in \mathcal{N}$. In addition we note that $R_{\widehat{\mu}_{n 1}}(z) \rightarrow z$ uniformly on every compact set in $\mathbb{C}^{+}$. Then, recalling the assumption of the theorem, we have $\sigma_{n} \rightarrow \sigma$ weakly on $[0, \infty]$ and $(4.54)$, and therefore we easily deduce from (4.53), that

$$
\frac{Z(z)}{z}=\exp \left\{\alpha+\int_{[0, \infty]} \frac{u+Z(z)}{1-u Z(z)} \sigma(d u)\right\}, \quad z \in \mathbb{C}^{+} .
$$

We see from (4.55) that $Z \not \equiv 0$. Since the function $Z(z)$ is univalent on $i \mathbb{C}^{+}$, it has a right inverse on the image $Z\left(i \mathbb{C}^{+}\right)$. Putting in $(4.55) z=Z^{(-1)}(w)$ and using (2.6), we conclude that $Z(z)=R_{\tau}(z)$, where $\tau$ is a $\bigotimes$-infinitely divisible p-measure. Hence $Z(z) \in \mathcal{K}$. In addition note that the equation (4.55) has an unique solution in class $\mathcal{K}$. Now suppose that $\left\{Z_{n}(z)\right\}$ does not converges to $Z(z)$ on some compact in $\mathbb{C}^{+}$. Then as above there exists a subsequence $\left\{n^{\prime \prime}\right\}$ such that $Z_{n^{\prime \prime}}(z) \rightarrow Z^{*}(z)$ as $n^{\prime \prime} \rightarrow \infty$ on every compact set in $\mathbb{C}^{+}$, where $Z^{*}(z) \in \mathcal{K}$. The function $Z^{*}(z)$ satisfies $Z^{*}(z) \not \equiv Z(z), z \in \mathbb{C}^{+}$. But $Z^{*}(z)$ is a solution of (4.55). We thus arrive at contradiction. Hence $\left\{Z_{n}(z)\right\}$ converges to $Z(z)$ uniformly on every compact set in $\mathbb{C}^{+}$. From (4.55) it follows that $Z(z)$ is infinitely divisible with parameters $(\alpha, \sigma(\{\infty\}), \sigma)$. Since $R_{\widehat{\mu}_{n 1}}\left(Z_{n}(z)\right) \rightarrow Z(z)$ uniformly on every compact set in $\mathbb{C}^{+}$, the sufficiency of the assumptions of the second part of the theorem is proved.

Necessity. First we assume without loss of generality that $\mu_{n k}(\{0\})=0, n \geq 1, k=$ $1, \ldots, k_{n}$, that is all $\mu_{n k} \in \mathcal{M}_{+}^{0}$. 
Indeed, by Proposition 3.2, we conclude from assumptions of the theorem that $\delta_{1 / a_{n}} \otimes$ $\tilde{\mu}_{n 1} \otimes \cdots \otimes \tilde{\mu}_{n k_{n}} \rightarrow \mu$ weakly as $n \rightarrow \infty$, where $\tilde{\mu}_{n k}:=\mu_{n k}-\mu_{n k}(\{0\})\left(\delta_{0}-\delta_{\gamma_{n k}}\right)$ and $\gamma_{n k}>0$ are sufficiently small positive numbers. It is clear that if prove the necessity conditions for the measures $\tilde{\mu}_{n k}$ we obtain the necessity conditions for the measures $\mu_{n k}$.

Denote $\mu_{n k}^{s}:=\mu_{n k} \otimes \bar{\mu}_{n k}, n \geq 1, k=1, \ldots, k_{n}$ and $\mu^{s}:=\mu \otimes \bar{\mu}$. By Proposition 3.2, we have the relation

$$
\mu^{(n, s)}:=\mu_{n 1}^{s} \otimes \mu_{n 2}^{s} \otimes \cdots \otimes \mu_{n k_{n}}^{s} \rightarrow \mu^{s} \quad \text { weakly as } n \rightarrow \infty .
$$

The relations (4.16) and (4.17) hold for the measures $\mu_{n k}^{s}, n \geq 1, k=1, \ldots, k_{n}$, with the functions $R_{\mu_{n k}^{s}}(z), n \geq 1, k=1, \ldots, k_{n}$, replacing $R_{\mu_{n k}}(z), n \geq 1, k=1, \ldots, k_{n}$, and with some functions $Z_{n k, s}(z) \in \mathcal{K}, n \geq 1, k=1, \ldots, k_{n}$, replacing $Z_{n k}(z), n \geq 1, k=$ $1, \ldots, k_{n}$. Rewrite (4.16) in the form

$$
\frac{R_{\mu_{n 1}^{s}}\left(Z_{n 1, s}(z)\right)}{z}=\frac{R_{\mu_{n 1}^{s}}\left(Z_{n 1, s}(z)\right)}{Z_{n 1, s}(z)} \ldots \frac{R_{\mu_{n k_{n}}^{s}}\left(Z_{n k_{n}, s}(z)\right)}{Z_{n k_{n}, s}(z)}, \quad z \in \mathbb{C}^{+} .
$$

By Proposition 3.5 and the relations (2.4) and (3.9), the measures $\mu_{n k}^{s}, n \geq 1, k=$ $1, \ldots, k_{n}$, are symmetric with respect to 1 , i.e., $\bar{\mu}_{n k}^{s}=\mu_{n k}^{s}$ and $\mu^{(n, s)}$ has the same property as well. Since $R_{\mu_{n k}^{s}}\left(Z_{n k, s}(z)\right)=R_{\mu^{(n, s)}}(z), z \in \mathbb{C}^{+}$, and, by Proposition 3.5, $\left|R_{\mu^{(n, s)}}\left(e^{i t}\right)\right|=$ $1,\left|R_{\mu_{n k}^{s}}\left(e^{i t}\right)\right|=1,0<t<2 \pi$, we conclude, using the univalence of $R$-functions in $\mathbb{C}^{+} \cap\left(i \mathbb{C}^{+}\right)$, that $\left|Z_{n k, s}\left(e^{i t}\right)\right|=1, n \geq 1, k=1, \ldots, k_{n}$, for the same $t$ as well.

Let us show that the p-measures $\mu_{n k}^{s}$ are infinitesimal as well. Since the estimates (4.15) hold for the functions $R_{\overline{\hat{\mu}}_{n k}}(z)$, we have the relation

$$
\log \Sigma_{\mu_{n k}^{s}}(z)=\log \Sigma_{\widehat{\mu}_{n k}}(z)+\log \Sigma_{\overline{\hat{\mu}}_{n k}}(z), \quad z \in D_{1}, n \geq n_{0}, \quad k=1, \ldots, k_{n},
$$

where we choose the principle branch of the logarithm. Using (4.15), it is not difficult to deduce from this relation that

$$
\left|R_{\mu_{n k}^{s}}(i)-i\right| \leq c(d) \varepsilon_{n k}, \quad n \geq n_{0}, k=1, \ldots, k_{n} .
$$

In view of (4.8) and (4.9), replacing the measures $\widehat{\mu}_{n k}$ by $\mu_{n k}^{s}$, and $\rho_{n k}$ by $\rho_{n k}^{s}$ (as defined in (4.2), replacing $\widehat{\mu}_{n k}$ by $\mu_{n k}^{s}$ ), we conclude

$$
\left|a\left(\rho_{n k}^{s}\right)-1\right| \leq c(d) \varepsilon_{n k} \quad \text { and } \quad \sigma^{2}\left(\rho_{n k}^{s}\right) \leq c(d) \varepsilon_{n k} .
$$

It remains to note that

$$
\begin{aligned}
\sigma^{2}\left(\rho_{n k}^{s}\right) & \geq \int_{\mathbb{R}_{+}} \frac{\left(u-a\left(\rho_{n k}^{s}\right)\right)^{2}}{1+u^{2}} \mu_{n k}^{s}(d u) \geq \int_{\mathbb{R}_{+}} \frac{(u-1)^{2}}{1+u^{2}} \mu_{n k}^{s}(d u) \\
& -2\left|a\left(\rho_{n k}^{s}\right)-1\right| \int_{\mathbb{R}_{+}} \frac{|u-1|}{1+u^{2}} \mu_{n k}^{s}(d u) \geq \int_{\mathbb{R}_{+}} \frac{(u-1)^{2}}{1+u^{2}} \mu_{n k}^{s}(d u)-2\left|a\left(\rho_{n k}^{s}\right)-1\right| .
\end{aligned}
$$


From (4.58) and (4.59) we see that the following inequality holds

$$
\varepsilon_{n k, s}:=\int_{\mathbb{R}_{+}} \frac{(u-1)^{2}}{1+u^{2}} \mu_{n k}^{s}(d u) \leq c(d) \varepsilon_{n k}, \quad n \geq n_{0}, k=1, \ldots, k_{n},
$$

which implies that the measures $\mu_{n k}^{s}$ are infinitesimal.

Hence $R_{\mu_{n k}^{s}}(z) \rightarrow z$ as $n \rightarrow \infty$ uniformly in $k=1, \ldots, k_{n}$ and on every compact set in $\mathbb{C} \backslash[0, \infty)$. Moreover $R_{\mu_{n k}^{s}}(z)$ is univalent in $\mathbb{C}^{+} \cap\left(i \mathbb{C}^{+}\right)$. Denote $z_{0}:=(-1+i) / \sqrt{2}$. Then $R_{\mu_{n k}^{s}}\left(z_{0}\right) \in \mathbb{C}^{+} \cap\left(i \mathbb{C}^{+}\right)$and $Z_{n k, s}\left(z_{0}\right) \in \mathbb{C}^{+} \cap\left(i \mathbb{C}^{+}\right)$. By the assumption of the theorem, we have $R_{\mu_{n k}^{s}}\left(Z_{n k, s}\left(z_{0}\right)\right) \rightarrow R_{\mu^{s}}\left(z_{0}\right)$ as $n \rightarrow \infty$ uniformly in $k=1, \ldots, k_{n}$. Therefore

$$
Z_{n k, s}\left(z_{0}\right) \rightarrow R_{\mu^{s}}\left(z_{0}\right)
$$

and

$$
\Re\left(R_{\mu_{n k}^{s}}\left(Z_{n k, s}\left(z_{0}\right)\right) / Z_{n k, s}\left(z_{0}\right)\right) \rightarrow 1
$$

as $n \rightarrow \infty$ uniformly in $k=1, \ldots, k_{n}$.

Using the integral representation

$$
R_{\mu_{n k}^{s}}(z)=c_{n k, s} z+z \int_{(0, \infty)} \frac{\tau_{n k, s}(d u)}{u-z}, \quad z \in \mathbb{C}^{+}, \quad n \geq 1, k=1, \ldots, k_{n},
$$

where $c_{n k, s} \geq 0$ and $\tau_{n k, s}$ are finite nonnegative measures such that

$$
\int_{(0, \infty)} \frac{\tau_{n k, s}(d u)}{1+u}<\infty
$$

we deduce

$$
\Im \frac{R_{\mu_{n k}^{s}}\left(Z_{n k, s}\left(z_{0}\right)\right)}{\left.Z_{n k, s}\left(z_{0}\right)\right)}=\Im Z_{n k, s}\left(z_{0}\right) \int_{(0, \infty)} \frac{\tau_{n k, s}(d u)}{\left(u-\Re Z_{n k, s}\left(z_{0}\right)\right)^{2}+\left(\Im Z_{n k, s}\left(z_{0}\right)\right)^{2}} .
$$

Since, as in (4.10), for $n \geq n_{0}, k=1, \ldots, k_{n}$,

$$
c_{16} \varepsilon_{n k, s} \leq \int_{(0, \infty)} \frac{\tau_{n k, s}(d u)}{1+u^{2}} \leq c_{17}(d) \varepsilon_{n k, s}
$$

we obtain from the previous formula, using (4.60),

$$
c_{18}\left(\mu^{s}\right) \varepsilon_{n k, s} \leq \Im \frac{R_{\mu_{n k}^{s}}\left(Z_{n k, s}\left(z_{0}\right)\right)}{Z_{n k, s}\left(z_{0}\right)} \leq c_{19}\left(\mu^{s}, d\right) \varepsilon_{n k, s}, \quad n \geq n_{0}, k=1, \ldots, k_{n} .
$$

We conclude from (4.61) and (4.63) that, for $n \geq n_{0}$ and $k=1, \ldots, k_{n}$,

$$
c_{20}\left(\mu^{s}\right) \varepsilon_{n k, s} \leq \arg \frac{R_{\mu_{n k}^{s}}\left(Z_{n k, s}\left(z_{0}\right)\right)}{Z_{n k, s}\left(z_{0}\right)} \leq c_{21}\left(\mu^{s}, d\right) \varepsilon_{n k, s} .
$$


In view of these inequalities and (4.57), we arrive at the relation

$$
\arg \frac{R_{\mu_{n 1}^{s}}\left(Z_{n 1, s}\left(z_{0}\right)\right)}{z_{0}}=\sum_{k=1}^{k_{n}} \arg \frac{R_{\mu_{n k}^{s}}\left(Z_{n k, s}\left(z_{0}\right)\right)}{Z_{n k, s}\left(z_{0}\right)} \geq c_{20}\left(\mu^{s}\right) \sum_{k=1}^{k_{n}} \varepsilon_{n k, s} .
$$

Therefore we obtain from (4.62) and (4.64) the relation

$$
\sum_{k=1}^{k_{n}} \int_{(0, \infty)} \frac{\tau_{n k, s}(d u)}{1+u^{2}} \leq c_{17}(d) \sum_{k=1}^{k_{n}} \varepsilon_{n k, s} \leq c\left(\mu^{s}\right), \quad n \geq n_{0} .
$$

Since $\mu_{n k}^{s}=\widehat{\mu}_{n k} \otimes \overline{\widehat{\mu}}_{n k}$, we note, by the definition of the free $\bigotimes$-convolution (see Section 3), that there exists a function $W_{n k}(z) \in \mathcal{K}$ such that $R_{\mu_{n k}^{s}}(z)=R_{\widehat{\mu}_{n k}}\left(W_{n k}(z)\right), z \in$ $\mathbb{C}^{+}$. The p-measures $\left\{\widehat{\mu}_{n k}\right\}$ and $\left\{\mu_{n k}^{s}\right\}$ are infinitesimal, therefore $R_{\mu_{n k}^{s}}(z)$ and $R_{\widehat{\mu}_{n k}}(z)$ tend to $z$ as $n \rightarrow \infty$ uniformly in $k=1, \ldots, k_{n}$ and every compact set in $\mathbb{C}^{+}$. Hence $W_{n k}\left(z_{0} \sqrt{2}\right) \rightarrow z_{0} \sqrt{2}$ as $n \rightarrow \infty$ uniformly in $k=1, \ldots, k_{n}$. In the following we shall use the relation

$$
\Im \frac{R_{\mu_{n k}^{s}}(z)}{z}=\Im \frac{R_{\widehat{\mu}_{n k}}\left(W_{n k}(z)\right)}{W_{n k}(z)} \Re \frac{W_{n k}(z)}{z}+\Re \frac{R_{\widehat{\mu}_{n k}}\left(W_{n k}(z)\right)}{W_{n k}(z)} \Im \frac{W_{n k}(z)}{z}, \quad z \in \mathbb{C}^{+} .
$$

Note that if $f(z) \in \mathcal{K}$, then $f(z) / z$ takes values in $\mathbb{C}^{+} \cap\left(-i \mathbb{C}^{+}\right)$when $z \in \mathbb{C}^{+} \cap\left(i \mathbb{C}^{+}\right)$. Therefore we conclude from the previous relation that

$$
\int_{(0, \infty)} \frac{\tau_{n k, s}(d u)}{u^{2}+1} \geq \Im \frac{R_{\mu_{n k}^{s}}\left(z_{0} \sqrt{2}\right)}{z_{0} \sqrt{2}} \geq \Im \frac{R_{\widehat{\mu}_{n k}}\left(W_{n k}\left(z_{0} \sqrt{2}\right)\right)}{W_{n k}\left(z_{0} \sqrt{2}\right)} \Re \frac{W_{n k}\left(z_{0} \sqrt{2}\right)}{z_{0} \sqrt{2}} \geq \frac{1}{4} \int_{(0, \infty)} \frac{\tau_{n k}(d u)}{u^{2}+1}
$$

for $n \geq n_{0}, k=1, \ldots, k_{n}$. In view of (4.10) and (4.65), we obtain from this that

$$
\frac{1}{32} \sum_{k=1}^{k_{n}} \varepsilon_{n k} \leq c_{17}(d) \sum_{k=1}^{k_{n}} \varepsilon_{n k, s} \leq c\left(\mu^{s}\right), \quad n \geq n_{0} .
$$

Return to (4.16) and (4.17). Since $R_{\mu^{(n)}}(z)=F_{\widehat{\mu}_{n}}\left(b_{n} z / a_{n}\right)$, where $b_{n}=\prod_{k=1}^{k_{n}} a_{n k}$, we see that $R_{\mu^{(n)}}(z)=R_{\widehat{\mu}_{n k}}\left(Z_{n k}\left(b_{n} z / a_{n}\right)\right), z \in \mathbb{C}^{+}, k=1, \ldots, k_{n}$. For $z \in \mathbb{C}^{+} \cap\left(i \mathbb{C}^{+}\right)$, functions from the class $\mathcal{K}$ take values in $\mathbb{C}^{+} \cap\left(i \mathbb{C}^{+}\right)$and are univalent. Since $R_{\widehat{\mu}_{n k}}(z)$ tend to $z$ and $R_{\widehat{\mu}_{n k}}\left(Z_{n k}\left(b_{n} z / a_{n}\right)\right)$ tend to $R_{\mu}(z)$ as $n \rightarrow \infty$ uniformly in $k=1, \ldots, k_{n}$ and on every compact set in $\mathbb{C}^{+}$, we obtain that the sequence $\left\{Z_{n k}\left(b_{n} z / a_{n}\right)\right\}_{n=1}^{\infty}$ converges uniformly in $k=1, \ldots, k_{n}$ and on every compact set in $\mathbb{C}^{+} \cap\left(i \mathbb{C}^{+}\right)$to the function $R_{\mu}(z) \in \mathcal{K}$. It is easy to see that this relation holds on every compact set in $\mathbb{C}^{+}$. Using relations (4.16) and (4.17) with $b_{n} z / a_{n}$ replacing $z$ and taking into account that the measures $\mu_{n 1}, \ldots, \mu_{n k_{n}}$ are infinitesimal and the upper bound (4.66) holds, we can repeat the arguments which we used for the proof of (4.48). We arrive at the relation, for $z \in \mathbb{C}^{+}$,

$$
\frac{Z_{n 1}\left(b_{n} z / a_{n}\right)}{b_{n} z / a_{n}}=\exp \left\{\int_{\mathbb{R}_{+}} \frac{u+1}{u-1} \sigma_{n}(d u)+\int_{\mathbb{R}_{+}} \frac{u+Z_{n 1}\left(b_{n} z / a_{n}\right)}{1-u Z_{n 1}\left(b_{n} z / a_{n}\right)} \sigma_{n}(d u)+\tilde{\Delta}_{n}(z)\right\},
$$


where $\tilde{\Delta}_{n}(z)$ is analytic in $\mathbb{C}^{+}$and $\tilde{\Delta}_{n}(z) \rightarrow 0$ on every compact set in $\mathbb{C}^{+}$. By $(4.66)$, the sequence $\left\{\sigma_{n}\right\}$ is tight in the vague topology. Therefore there exists a subsequence $\left\{n^{\prime}\right\}$ such that $\left\{\sigma_{n^{\prime}}\right\}$ converges to some finite nonnegative measure $\sigma$ in the vague topology and $\lim _{n^{\prime} \rightarrow \infty} \sigma_{n^{\prime}}\left(\mathbb{R}_{+}\right)$exists and finite. Thus, we can conclude from (4.67) that $b_{n}^{\prime} d_{n}^{\prime} / a_{n}^{\prime} \rightarrow e^{\alpha_{1}^{\prime}}$ as $n^{\prime} \rightarrow \infty$, where $\alpha_{1}^{\prime} \in \mathbb{R}$ and

$$
d_{n}:=\exp \left\{\int_{\mathbb{R}_{+}} \frac{u+1}{u-1} \sigma_{n}(d u)\right\},
$$

and the following relation holds

$$
\frac{R_{\mu}(z)}{z}=\exp \left\{\alpha_{1}^{\prime}-\frac{\alpha_{2}^{\prime}}{R_{\mu}(z)}+\int_{\mathbb{R}_{+}} \frac{u+R_{\mu}(z)}{1-u R_{\mu}(z)} \sigma(d u)\right\}, \quad z \in \mathbb{C}^{+},
$$

with $\alpha_{2}^{\prime} \geq 0$.

We shall show that $\left\{\sigma_{n}\right\}$ converges to a measure $\sigma$ on $\mathbb{R}_{+}$in the vague topology. Assume to the contrary that there exists a subsequence $\left\{n^{\prime \prime}\right\}$ such that $\left\{\sigma_{n^{\prime \prime}}\right\}$ converges in the vague topology to some finite nonnegative measure $\sigma_{1} \not \equiv \sigma$ and $\lim _{n^{\prime \prime} \rightarrow \infty} \sigma_{n^{\prime \prime}}\left(\mathbb{R}_{+}\right)$ exists and finite. Then $b_{n}^{\prime \prime} d_{n}^{\prime \prime} / a_{n}^{\prime \prime} \rightarrow e^{\alpha_{1}^{\prime \prime}}$ as $n^{\prime \prime} \rightarrow \infty$, where $\alpha_{1}^{\prime \prime} \in \mathbb{R}$, and (4.68) holds with $\alpha_{1}^{\prime \prime} \in \mathbb{R}, \alpha_{2}^{\prime \prime} \geq 0$ replacing $\alpha_{1}^{\prime}, \alpha_{2}^{\prime}$ and $\sigma_{1}$ replacing $\sigma$. Comparing the relations (4.68) with old and new parameters, we deduce the formula

$\alpha_{1}^{\prime}-\frac{\alpha_{2}^{\prime}}{z}+\alpha_{3}^{\prime} z+\int_{(0, \infty)} \frac{u+z}{1-u z} \sigma(d u)=\alpha_{1}^{\prime \prime}+2 m \pi i-\frac{\alpha_{2}^{\prime \prime}}{z}+\alpha_{3}^{\prime \prime} z+\int_{(0, \infty)} \frac{u+z}{1-u z} \sigma_{1}(d u), \quad z \in \mathbb{C}^{+}$,

where $m \in \mathbb{Z}$ and $0 \leq \alpha_{3}^{\prime}<\infty, 0 \leq \alpha_{3}^{\prime \prime}<\infty$. By (3.3), we easily conclude that

$$
\left|\int_{(0, \infty)} \frac{u+z}{1-u z} \sigma(d u)\right|+\left|\int_{(0, \infty)} \frac{u+z}{1-u z} \sigma_{1}(d u)\right|=o(|z|), \quad z \in \mathbb{C}^{+}
$$

as $z \rightarrow 0$ or $z \rightarrow \infty$ nontangentially. Comparing the behavior of all terms in (4.69) as $z \rightarrow \infty$ and as $z \rightarrow 0$, we easily see that $\alpha_{j}^{\prime}=\alpha_{j}^{\prime \prime}$ for $j=2,3$. Applying the StieltjesPerron inversion formula (see Section 3), we obtain that $\sigma=\sigma_{1}$ and $m=0$. Finally we get $\alpha_{1}^{\prime}=\alpha_{1}^{\prime \prime}$, a contradiction.

Since $\left\{\sigma_{n}\right\}$ converges to the measure $\sigma$ in the vague topology, we obtain from (4.67) that $a_{n} /\left(b_{n} d_{n}\right) \rightarrow e^{\alpha_{1}^{\prime}}$ as $n \rightarrow \infty$. It remains to show that $\left\{\sigma_{n}\right\}$ converges to the measure $\sigma$ weakly in $[0, \infty]$. For this we note that it follows from (4.67) and (4.68) the relation

$$
\lim _{n \rightarrow \infty} \int_{[0, \infty]}\left(\frac{1+R_{\mu}^{2}(z)}{1-u R_{\mu}(z)}-1\right) \sigma_{n}(d u)=\int_{[0, \infty]}\left(\frac{1+R_{\mu}^{2}(z)}{1-u R_{\mu}(z)}-1\right) \sigma(d u)
$$

holds on every compact set in $\mathbb{C}^{+}$, where $\sigma(\{\infty\})=\alpha_{2}^{\prime}$. This relation implies that $\lim _{n \rightarrow \infty} \sigma_{n}([0, \infty])=\sigma([0, \infty])$ as was to be proved. 
Thus the necessity of the assumptions of the theorem is proved and Theorem 2.1 is completely proved.

\section{Multiplicative fRee limit theorem in $\mathcal{M}_{*}$}

In Section 5 we prove Theorem 2.3 and Theorem 2.4. Here we denote by $c$ positive absolute constants. For some measure $\nu$ we denote by $c(\nu)$ positive constants which depend on the measure $\nu$. By $c_{j}, c_{j}(\nu), j=1, \ldots$, we denote explicit absolute positive constants and explicit positive constants depending on the corresponding measure, respectively.

Let $\left\{\mu_{n k}: n \geq 1,1 \leq k \leq k_{n}\right\}$, where $k_{n} \uparrow \infty$ as $n \rightarrow \infty$, be a triangular scheme of measures in $\mathcal{M}_{*}$. It is not difficult to see that condition (2.16) is equivalent to the following relation

$$
\max _{k=1, \ldots, k_{n}} \int_{[-\pi, \pi)}(1-\cos y) \mu_{n k}(d y) \rightarrow 0, \quad n \rightarrow \infty .
$$

Recall that $\widehat{\mu}_{n k}$ are p-measures such that $\widehat{\mu}_{n k}([-\pi, x))=\mu_{n k}\left(\left[-\pi+a_{n k}, x+a_{n k}\right)\right)$, where $a_{n k}:=\int_{[-\pi, \pi)} \sin u \mu_{n k}(d u)$. Since $\max _{k=1, \ldots, k_{n}}\left|a_{k n}\right| \rightarrow 0$ as $n \rightarrow \infty$, we conclude

$$
\varepsilon_{n}:=\max _{k=1, \ldots, k_{n}} \varepsilon_{n k}:=\max _{k=1, \ldots, k_{n}} \int_{[-\pi, \pi)}(1-\cos y) \widehat{\mu}_{n k}(d y) \rightarrow 0, \quad n \rightarrow \infty .
$$

Proof of Theorem 2.3. Sufficiency. From Proposition 3.7 we obtain, for all $z \in \mathbb{D}$,

$$
\frac{Q_{\widehat{\mu}_{n 1}}\left(Z_{n 1}(z)\right)}{z}=\frac{Q_{\widehat{\mu}_{n 1}}\left(Z_{n 1}(z)\right)}{Z_{n 1}(z)} \ldots \frac{Q_{\widehat{\mu}_{n k_{n}}}\left(Z_{n k_{n}}(z)\right)}{Z_{n k_{n}}(z)}
$$

where $Z_{n 1}(z), \ldots, Z_{n k_{n}}(z)$ belong to the class $\mathcal{S}_{*}$, and

$$
Q_{\widehat{\mu}_{n 1}}\left(Z_{n 1}(z)\right)=\cdots=Q_{\widehat{\mu}_{n k_{n}}}\left(Z_{n k_{n}}(z)\right) .
$$

Moreover, $Q_{n}(z):=Q_{\widehat{\mu}_{n}}(z)=Q_{\widehat{\mu}_{n 1}}\left(Z_{n 1}(z)\right)$, where $\widehat{\mu}_{n}:=\widehat{\mu}_{n 1} \otimes \cdots \otimes \widehat{\mu}_{n k_{n}}$. Taking into account (2.10) we have

$$
Q_{\widehat{\mu}_{n k}}(z)=\frac{F_{\widehat{\mu}_{n k}}(z)-1}{F_{\widehat{\mu}_{n k}}(z)+1}, \quad z \in \mathbb{D}, \quad k=1, \ldots, k_{n},
$$

where the functions $F_{\widehat{\mu}_{n k}}(z)$ admit the representation (2.11) with some p-measures $\tilde{\sigma}_{n k}$ replacing $\sigma$.

In the sequel we denote $S_{\mu}(z):=Q_{\mu}(z) / z, z \in \mathbb{D}$, for $\mu \in \mathcal{M}_{*}$.

Consider the functions $S_{\widehat{\mu}_{n k}}(z):=Q_{\widehat{\mu}_{n k}}(z) / z, n \geq 1, k=1, \ldots, k_{n}$, in $\mathbb{D}$. By Schwarz's lemma, these functions belong to the class $\mathcal{S}$ (the class of Schur functions) and

$$
S_{\widehat{\mu}_{n k}}(0)=\int_{[-\pi, \pi)} e^{i y} \widehat{\mu}_{n k}(d y)=e^{-i a_{n k}} \int_{[-\pi, \pi)} e^{i y} \mu_{n k}(d y) .
$$

It is not difficult to verify that

$$
\left|1-S_{\widehat{\mu}_{n k}}(0)\right| \leq c \varepsilon_{n k}
$$


for $n \geq n_{0}, k=1, \ldots, k_{n}$, with a sufficienly large positive constant $n_{0}$. Indeed, we easily have

$$
\Im S_{\widehat{\mu}_{n k}}(0)=\int_{[-\pi, \pi)} \sin y \widehat{\mu}_{n k}(d y)=\int_{[-\pi, \pi)}\left(\sin y-a_{n k}\right) \mu_{n k}+c \theta \varepsilon_{n k}=c \theta \varepsilon_{n k}
$$

Since $\Re\left(1-S_{\widehat{\mu}_{n k}}(z)\right) \geq 0$, we see that $1-S_{\widehat{\mu}_{n k}}(z)$ belongs to Carathéodory's class $\mathcal{C}$. Therefore (see Section 3), this function admits the representation

$$
1-S_{\widehat{\mu}_{n k}}(z)=-i \Im S_{\widehat{\mu}_{n k}}(0)+\int_{[-\pi, \pi)} \frac{e^{i y}+z}{e^{i y}-z} \sigma_{n k}(d y), \quad z \in \mathbb{D},
$$

where $\sigma_{n k}$ is a finite nonnegative measure such that $\sigma_{n k}([-\pi, \pi))=1-\Re S_{\widehat{\mu}_{n k}}(0)$. By (5.5), we note that $\sigma_{n k}([-\pi, \pi)) \leq c \varepsilon_{n k}$. Therefore we conclude

$$
\left|1-S_{\widehat{\mu}_{n k}}(z)\right| \leq c \varepsilon_{n k}+\int_{[-\pi, \pi)} \frac{1+|z|}{1-|z|} \sigma_{n k}(d y) \leq \frac{c \varepsilon_{n k}}{1-|z|}, \quad z \in \mathbb{D} .
$$

In addition we have from (5.6), for $z_{1}, z_{2} \in \mathbb{D}$,

$$
\left|S_{\widehat{\mu}_{n k}}\left(z_{1}\right)-S_{\widehat{\mu}_{n k}}\left(z_{2}\right)\right| \leq \int_{[-\pi, \pi)} \frac{2\left|z_{1}-z_{2}\right|}{\left|e^{i y}-z_{1}\right|\left|e^{i y}-z_{2}\right|} \sigma_{n k}(d y) \leq \frac{c \varepsilon_{n k}\left|z_{1}-z_{2}\right|}{\left(1-\left|z_{1}\right|\right)\left(1-\left|z_{2}\right|\right)} .
$$

Return to the functions $Z_{n k}(z)$ in (5.2) and (5.3). These functions are in the class $\mathcal{S}_{*}$. Therefore

$$
\left|Z_{n k}(z) / z\right| \leq 1, \quad z \in \mathbb{D} .
$$

Using (5.7) and (5.9) we obtain, for $z \in \mathbb{D}$,

$$
\left|1-S_{\widehat{\mu}_{n k}}\left(Z_{n k}(z)\right)\right| \leq \frac{c \varepsilon_{n k}}{1-\left|Z_{n k}(z)\right|} \leq \frac{c \varepsilon_{n k}}{1-|z|}
$$

Let $r_{n}:=1-c_{1} \varepsilon_{n}, n \geq n_{0}$, with sufficiently large positive constants $c_{1}$ and $n_{0}$. Then, by $(5.10),\left|1-S_{\widehat{\mu}_{n k}}\left(Z_{n k}(z)\right)\right| \leq c \varepsilon_{n k} /\left(1-r_{n}\right) \leq 1 / 2$ for $z \in \mathbb{D}_{r_{n}}:=\left\{z \in \mathbb{C}:|z|<r_{n}\right\}$. In view of (5.10), we obtain, using the series expansion for $\log (1+z),|z|<1$,

$$
S_{\widehat{\mu}_{n k}}\left(Z_{n k}(z)\right)=\exp \left\{-\left(1-S_{\widehat{\mu}_{n k}}\left(Z_{n k}(z)\right)\right)+q_{n k}(z)\right\}, \quad z \in \mathbb{D}_{r_{n}},
$$

where the analytic function $q_{n k}(z)$ in $\mathbb{D}_{r_{n}}$ admits the estimate $\left|q_{n k}(z)\right| \leq c \varepsilon_{n k}^{2} /(1-|z|)^{2}, z \in$ $\mathbb{D}_{r_{n}}$.

In the next step we conclude from (5.8) and (5.9) that, for $z \in \mathbb{D}$,

$$
\left|S_{\widehat{\mu}_{n k}}\left(Z_{n k}(z)\right)-S_{\widehat{\mu}_{n k}}\left(Z_{n 1}(z)\right)\right| \leq \frac{c \varepsilon_{n k}\left|Z_{n k}(z)-Z_{n 1}(z)\right|}{\left(1-\left|Z_{n k}(z)\right|\right)\left(1-\left|Z_{n 1}(z)\right|\right)} \leq \frac{c \varepsilon_{n k}\left|Z_{n k}(z)-Z_{n 1}(z)\right|}{(1-|z|)^{2}} .
$$


On the other hand we see from (5.3) and (5.10) that, for $z \in \mathbb{D}_{r_{n}}$,

$$
\begin{aligned}
\left|\frac{Z_{n k}(z)}{Z_{n 1}(z)}-1\right| & =\left|\frac{S_{\widehat{\mu}_{n k}}\left(Z_{n 1}(z)\right)}{S_{\widehat{\mu}_{n k}}\left(Z_{n k}(z)\right)}-1\right| \\
& \leq 2\left|1-S_{\widehat{\mu}_{n k}}\left(Z_{n k}(z)\right)\right|+2\left|1-S_{\widehat{\mu}_{n k}}\left(Z_{n 1}(z)\right)\right| \leq \frac{c \varepsilon_{n k}}{1-|z|} .
\end{aligned}
$$

Hence

$$
\left|Z_{n k}(z)-Z_{n 1}(z)\right| \leq \frac{c \varepsilon_{n k}|z|}{1-|z|}, \quad z \in \mathbb{D}_{r_{n}}
$$

Applying this inequality to (5.12) we obtain

$$
\left|g_{n k}(z)\right| \leq \frac{c \varepsilon_{n k}^{2}|z|}{(1-|z|)^{3}} \quad \text { where } \quad g_{n k}(z):=S_{\widehat{\mu}_{n k}}\left(Z_{n k}(z)\right)-S_{\widehat{\mu}_{n k}}\left(Z_{n 1}(z)\right) .
$$

for $z \in \mathbb{D}_{r_{n}}$ and $n \geq n_{0}, k=1, \ldots, k_{n}$.

Note that, for $z \in \mathbb{D}$,

$$
\begin{aligned}
(1-z)(1 & \left.+\psi_{\widehat{\mu}_{n k}}(z)\right)-1=-\int_{[-\pi, \pi)} \frac{z\left(1-e^{i y}\right)}{1-z e^{i y}} \widehat{\mu}_{n k}(d y) \\
= & -\sum_{k=0}^{\infty} z^{k+1} \int_{[-\pi, \pi)} e^{i k y}\left(1-e^{i y}\right) \widehat{\mu}_{n k}(d y)=-\sum_{p=0}^{\infty} z^{p+1} \int_{[-\pi, \pi)}\left(1-e^{i y}\right) \widehat{\mu}_{n k}(d y) \\
& +\sum_{p=0}^{\infty} z^{p+1} \int_{[-\pi, \pi)}\left(1-e^{i p y}\right)\left(1-e^{i y}\right) \widehat{\mu}_{n k}(d y) .
\end{aligned}
$$

Using (5.5) and the simple relation $\int_{[-\pi, \pi)}\left|1-e^{i y}\right|^{2} \widehat{\mu}_{n k}(d y) \mid=2 \int_{[-\pi, \pi)}(1-\cos y) \widehat{\mu}_{n k}(y)=$ $2 \varepsilon_{n k}$, we easily arrive at the upper bound, for $z \in \mathbb{D}_{r_{n}}$,

$$
\begin{gathered}
\left|(1-z)\left(1+\psi_{\widehat{\mu}_{n k}}(z)\right)-1\right| \leq \frac{2|z|}{1-|z|} \int_{[-\pi, \pi)}\left(1-e^{i y}\right) \widehat{\mu}_{n k}(d y) \\
+\sum_{p=1}^{\infty} p|z|^{p+1} \int_{[-\pi, \pi)}\left|1-e^{i y}\right|^{2} \widehat{\mu}_{n k}(d y) \leq c \varepsilon_{n k}\left(\frac{|z|}{1-|z|}+\frac{|z|^{2}}{(1-|z|)^{2}}\right) \leq c \varepsilon_{n k} \frac{|z|}{(1-|z|)^{2}} .
\end{gathered}
$$

On the other hand

$$
1-(1-z) \frac{\psi_{\widehat{\mu}_{n k}}(z)}{z}=\int_{[-\pi, \pi)} \frac{1-e^{i y}}{1-z e^{i y}} \widehat{\mu}_{n k}(d y) .
$$

Repeating the previous arguments we obtain the inequality

$$
\left|1-(1-z) \frac{\psi_{\widehat{\mu}_{n k}}(z)}{z}\right| \leq c \varepsilon_{n k} \frac{1}{(1-|z|)^{2}}, \quad z \in \mathbb{D}_{r_{n}} .
$$


Denote $r_{n}^{\prime}=1-c_{2} \varepsilon_{n}, n \geq n_{0}$, with a sufficiently large constant $c_{2}>c_{1}$.

By (5.14) and (5.15) and the series expansion of the function $1 /(1+z)$ for $z \in \mathbb{D}$, we have, for $z \in \mathbb{D}_{r_{n}^{\prime}}$,

$$
\begin{aligned}
1-S_{\widehat{\mu}_{n k}}(z) & =1-\frac{\psi_{\widehat{\mu}_{n k}}(z)}{z\left(1+\psi_{\widehat{\mu}_{n k}}(z)\right)}=\frac{(1-z)\left(1-\frac{1-z}{z} \psi_{\widehat{\mu}_{n k}}(z)\right)}{(1-z)\left(1+\psi_{\widehat{\mu}_{n k}}(z)\right.} \\
& =f_{n k}(z)+d_{n k}(z):=\int_{[-\pi, \pi)} \frac{(1-z)\left(1-e^{i y}\right)}{1-z e^{i y}} \widehat{\mu}_{n k}(d y)+d_{n k}(z) \\
& =-i \int_{[-\pi, \pi)} \sin y \widehat{\mu}_{n k}(d y)+\int_{[-\pi, \pi)} \frac{1+z e^{i y}}{1-z e^{i y}}(1-\cos y) \widehat{\mu}_{n k}(d y)+d_{n k}(z),
\end{aligned}
$$

where $d_{n k}(z)$ is an analytic function in $\mathbb{D}_{r_{n}^{\prime}}$ such that $\left|d_{n k}(z)\right| \leq c \varepsilon_{n k}^{2} /(1-|z|)^{4}$. It remains to note, by (5.9), that from (5.16) it follows

$$
1-S_{\widehat{\mu}_{n k}}\left(Z_{n 1}(z)\right)=f_{n k}\left(Z_{n 1}(z)\right)+b_{n k}(z)
$$

for $z \in \mathbb{D}_{r_{n}^{\prime}}$ and $n \geq n_{0}, k=1, \ldots, k_{n}$, where $b_{n k}(z)$ is analytic function in $z \in \mathbb{D}_{r_{n}^{\prime}}$ such that $\left|b_{n k}(z)\right| \leq c \varepsilon_{n k}^{2} \mid /(1-|z|)^{4}$.

Using (5.11), (5.13), and (5.17), we rewrite (5.2) in the form

$$
\begin{aligned}
& \frac{Q_{\widehat{\mu}_{n 1}}\left(Z_{n 1}(z)\right)}{z}=\exp \left\{-\sum_{k=1}^{k_{n}} f_{n k}\left(Z_{n 1}(z)\right)+\sum_{k=1}^{k_{n}} g_{n k}(z)+\sum_{k=1}^{k_{n}} q_{n k}(z)-\sum_{k=1}^{k_{n}} b_{n k}(z)\right\} \\
& :=\exp \left\{i \sum_{k=1}^{k_{n}} \int_{[-\pi, \pi)} \sin y \widehat{\mu}_{n k}(d y)-\int_{[-\pi, \pi)} \frac{1+Z_{n 1}(z) e^{i y}}{1-Z_{n 1}(z) e^{i y}} \nu_{n}(d y)+g_{n}(z)+q_{n}(z)-b_{n}(z)\right\},
\end{aligned}
$$

where, for sufficiently large $n \geq n_{0}$,

$$
\begin{aligned}
\left|g_{n}(z)\right|+\left|q_{n}(z)\right|+\left|b_{n}(z)\right| & \leq \sum_{k=1}^{k_{n}}\left|g_{n k}(z)\right|+\sum_{k=1}^{k_{n}}\left|q_{n k}(z)\right|+\sum_{k=1}^{k_{n}}\left|b_{n k}(z)\right| \\
& \leq \frac{c \varepsilon_{n}}{(1-|z|)^{4}} \sum_{k=1}^{k_{n}} \varepsilon_{n k} \leq \frac{c(\nu([-\pi, \pi))+1) \varepsilon_{n}}{(1-|z|)^{4}}
\end{aligned}
$$

for $z \in \mathbb{D}_{r_{n}^{\prime}}$. From (5.19) we see that, for $n \geq n_{0}$,

$$
\left|g_{n}(z)\right|+\left|q_{n}(z)\right|+\left|b_{n}(z)\right| \leq c(\nu([-\pi, \pi))+1) \varepsilon_{n}^{1 / 5}, \quad z \in \mathbb{D}_{r_{n}^{\prime \prime}},
$$

where $r_{n}^{\prime \prime}:=1-\varepsilon_{n}^{1 / 5}$. Denote $\beta_{n}:=\sum_{k=1}^{k_{n}} a_{n k}-a_{n}$.

Consider the sequence of the functions $\left\{\tilde{Z}_{n 1}(z):=Z_{n 1}\left(z e^{i \beta_{n}}\right)\right\}_{n=1}^{\infty}, z \in \mathbb{D}$. By (5.9), we conclude that there exists a subsequence $\left\{n^{\prime}\right\}$ such that $\tilde{Z}_{n^{\prime} 1} \rightarrow Z(z)$ as $n^{\prime} \rightarrow \infty$ uniformly on every compact set in $\mathbb{D}$ and $Z(z) \in \mathcal{S}$. In addition we note that $Q_{\widehat{\mu}_{n 1}}(z) \rightarrow z$ uniformly 
on every compact set in $\mathbb{D}$. Then, by the assumption of the theorem that $\nu_{n} \rightarrow \nu$ weakly, $\alpha_{n}-a_{n} \rightarrow \alpha, \bmod 2 \pi$, and (5.20), we deduce from (5.18) the relation

$$
\frac{Z(z)}{z}=\exp \left\{i \alpha-\int_{[-\pi, \pi)} \frac{1+Z(z) e^{i y}}{1-Z(z) e^{i y}} \nu(d y)\right\}, \quad z \in \mathbb{D},
$$

where $\alpha \in[-\pi, \pi)$. This implies that $Z^{\prime}(0) \neq 0$. Hence $Z(z) \in \mathcal{S}_{*}$.

Since $Z(z)$ has an inverse $Z^{(-1)}(z)$ defined in some neighborhood of 0 , we see that the equation (5.21) has an unique solution in the class of functions $Z \in \mathcal{S}_{*}$. Now assume that $\left\{\tilde{Z}_{n 1}(z)\right\}_{n=1}^{\infty}$ does not converge to $Z(z)$ on some compact set in $\mathbb{D}$. Then, as above, there exists a subsequence $\left\{n^{\prime \prime}\right\}$ such that $\tilde{Z}_{n^{\prime \prime} 1}(z) \rightarrow Z^{*}(z)$ as $n^{\prime \prime} \rightarrow \infty$ on every compact set in $\mathbb{D}$, and $Z^{*}(z) \in \mathcal{S}_{*}$ and $Z(z) \not \equiv Z^{*}(z), z \in \mathbb{D}$. But $Z^{*}(z)$ is a solution of (5.21) and we arrive at a contradiction. Hence, $\left\{\tilde{Z}_{n 1}(z)\right\}$ converges to $Z(z)$ uniformly on every compact set in $\mathbb{D}$. Using (5.21) we note that $Z(z)$ is infinitely divisible with the parameters $(\alpha, \nu)$. Since $Q_{\widehat{\mu}_{n k}}\left(\tilde{Z}_{n 1}(z)\right) \rightarrow Z(z)$ on every compact set in $\mathbb{D}$, the sufficiency of the assumptions of Theorem 2.3 is proved.

Necessity. Denote $\mu_{n k}^{s}:=\mu_{n k} \otimes \bar{\mu}_{n k}, n \geq 1, k=1, \ldots, k_{n}$ and $\mu^{s}:=\mu \otimes \bar{\mu}$. By Proposition 3.2, we have the relation

$$
\mu^{(n, s)}:=\mu_{n 1}^{s} \otimes \mu_{n 2}^{s} \otimes \cdots \otimes \mu_{n k_{n}}^{s} \rightarrow \mu^{s} \quad \text { weakly as } n \rightarrow \infty .
$$

For the measures $\mu_{n k}^{s}, n \geq 1, k=1, \ldots, k_{n}$, the relations (5.2) and (5.3) hold with the functions $Q_{\mu_{n k}^{s}}(z), n \geq 1, k=1, \ldots, k_{n}$, replacing $Q_{\mu_{n k}}(z), n \geq 1, k=1, \ldots, k_{n}$, and with some functions $Z_{n k, s}(z) \in \mathcal{S}_{*}, n \geq 1, k=1, \ldots, k_{n}$, replacing $Z_{n k}(z), n \geq 1, k=$ $1, \ldots, k_{n}$. Rewrite (5.2) in the form

$$
\frac{Q_{\mu^{(n, s)}}(z)}{z}=\frac{Q_{\mu_{n 1}^{s}}\left(Z_{n 1, s}(z)\right)}{Z_{n 1, s}(z)} \ldots \frac{Q_{\mu_{n k_{n}}^{s}}\left(Z_{n k_{n}, s}(z)\right)}{Z_{n k_{n}, s}(z)}, \quad z \in \mathbb{D} .
$$

By Proposition 3.8, the measures $\mu_{n k}^{s}, n \geq 1, k=1, \ldots, k_{n}$, are symmetric, i.e., $\bar{\mu}_{n k}^{s}=\mu_{n k}^{s}$ and $\mu^{(n, s)}$ has the same property as well. Since $Q_{\mu_{n k}^{s}}\left(Z_{n k, s}(z)\right)=Q_{\mu^{(n, s)}}(z), z \in \mathbb{D}$, and by Proposition 3.8, $\Im Q_{\mu^{(n, s)}}(t)=0, \Im Q_{\mu_{n k}^{s}}(t)=0,-1<t<1$, we conclude that, for the same $t, \Im Z_{n k, s}(t)=0, n \geq 1, k=1, \ldots, k_{n}$, as well.

Let us show that the p-measures $\mu_{n k}^{s}$ are infinitesimal. Consider the functions $S_{\mu_{n k}^{s}}(z)$, $n \geq 1, k=1, \ldots, k_{n}$, in $\mathbb{D}$. Since $\mu_{n k}^{s}=\widehat{\mu}_{n k} \otimes \overline{\widehat{\mu}}_{n k}$, we note, by the definition of the free $\nabla$ convolution (see Section 3), that there exists functions $W_{n k, 1}(z), W_{n k, 2}(z) \in \mathcal{S}_{*}$ such that $Q_{\mu_{n k}^{s}}(z)=Q_{\widehat{\mu}_{n k}}\left(W_{n k, 1}(z)\right)$ and $S_{\mu_{n k}^{s}}(z)=S_{\widehat{\mu}_{n k}}\left(W_{n k, 1}(z)\right) S_{\bar{\mu}_{n k}}\left(W_{n k, 2}(z)\right), z \in \mathbb{D}$. Choosing in the last relation $z=0$ we get $S_{\mu_{n k}^{s}}(0)=S_{\widehat{\mu}_{n k}}(0) S_{\bar{\mu}_{n k}}(0)=\left|S_{\widehat{\mu}_{n k}}(0)\right|^{2}$. Therefore

$$
\varepsilon_{n k, s}:=1-S_{\mu_{n k}^{s}}(0)=\int_{[-\pi, \pi)}(1-\cos y) \mu_{n k}^{s}(d y) \rightarrow 0
$$

as $n \rightarrow \infty$ uniformly in $k=1, \ldots, k_{n}$ as was to be proved. 
Note that (5.23) implies the relation

$$
S_{\mu^{(n, s)}}(0)=S_{\mu_{n 1}^{s}}(0) S_{\mu_{n 2}^{s}}(0) \ldots S_{\mu_{n k_{n}}^{s}}(0)
$$

from which we conclude with the help of (5.24)

$$
S_{\mu^{(n, s)}}(0)=\exp \left\{-\left(1+c \theta \max _{k=1, \ldots, k_{n}} \varepsilon_{n k, s}\right) \sum_{k=1}^{k_{n}} \varepsilon_{n k, s}\right\}, \quad n \geq n_{0} .
$$

By the assumption of the theorem, we have $S_{\mu^{(n, s)}}(0) \rightarrow S_{\mu^{s}}(0) \neq 0$ and we obtain from $(5.25)$

$$
\sum_{k=1}^{k_{n}} \varepsilon_{n k, s}<c\left(\mu^{s}\right)<\infty
$$

Since $\mu_{n k}^{s}=\widehat{\mu}_{n k} \otimes \overline{\widehat{\mu}}_{n k}$, we saw above that there exist functions $W_{n k, 1}(z) \in \mathcal{S}_{*}$ such that $Q_{\mu_{n k}^{s}}(z)=Q_{\widehat{\mu}_{n k}}\left(W_{n k, 1}(z)\right), z \in \mathbb{D}$. The p-measures $\left\{\widehat{\mu}_{n k}\right\}$ and $\left\{\mu_{n k}^{s}\right\}$ are infinitesimal, therefore $Q_{\mu_{n k}^{s}}(z)$ and $Q_{\widehat{\mu}_{n k}}(z)$ tend to $z$ as $n \rightarrow \infty$ uniformly in $k=1, \ldots, k_{n}$ and on every compact set in $\mathbb{D}$. Thus $W_{n k}(z) \rightarrow z$ as $n \rightarrow \infty$ uniformly in $k=1, \ldots, k_{n}$ and on every compact set in $\mathbb{D}$. Therefore we have $W_{n k}^{\prime}(0) \rightarrow 1$ as $n \rightarrow \infty$ uniformly in $k=1, \ldots, k_{n}$. In addition, by (5.9), the functions $W_{n k}(z)$ satisfy $\left|W_{n k}^{\prime}(0)\right| \leq 1, n \geq 1, k=1, \ldots, k_{n}$.

The relation $S_{\mu_{n k}^{s}}(0)=S_{\widehat{\mu}_{n k}}(0) W_{n k, 1}^{\prime}(0)$ implies

$$
1-S_{\mu_{n k}^{s}}(0)=1-\Re S_{\widehat{\mu}_{n k}}(0)+\Re S_{\widehat{\mu}_{n k}}(0)\left(1-\Re W_{n k, 1}^{\prime}(0)\right)+\Im S_{\widehat{\mu}_{n k}}(0) \Im W_{n k, 1}^{\prime}(0)
$$

and

$$
0=\Im S_{\widehat{\mu}_{n k}}(0) \Re W_{n k, 1}^{\prime}(0)+\Re S_{\widehat{\mu}_{n k}}(0) \Im W_{n k, 1}^{\prime}(0) .
$$

From the last two relations we easily obtain

$$
1-S_{\mu_{n k}^{s}}(0)=1-\Re S_{\widehat{\mu}_{n k}}(0)+\Re S_{\widehat{\mu}_{n k}}(0)\left(1-\Re W_{n k, 1}^{\prime}(0)\left(1+\left(\frac{\Im S_{\widehat{\mu}_{n k}}(0)}{\Re S_{\widehat{\mu}_{n k}}(0)}\right)^{2}\right) .\right.
$$

From (5.5) it follows that

$$
1-\Re S_{\widehat{\mu}_{n k}}(0)=\varepsilon_{n k}, \quad\left|\Im S_{\widehat{\mu}_{n k}}(0)\right| \leq c \varepsilon_{n k}, \quad n \geq n_{0}, \quad k=1, \ldots, k_{n} .
$$

Applying (5.28) to (5.27), we deduce

$$
\begin{aligned}
\varepsilon_{n k}=1-\Re S_{\widehat{\mu}_{n k}}(0) & \leq 1-S_{\mu_{n k}^{s}}(0)+\Re S_{\widehat{\mu}_{n k}}(0) \Re W_{n k, 1}^{\prime}(0)\left(\frac{\Im S_{\widehat{\mu}_{n k}}(0)}{\Re S_{\widehat{\mu}_{n k}}(0)}\right)^{2} \\
& \leq 1-S_{\mu_{n k}^{s}}(0)+c \varepsilon_{n k}^{2}=\varepsilon_{n k, s}+c \varepsilon_{n k}^{2}
\end{aligned}
$$

for $n \geq n_{0}, k=1, \ldots, k_{n}$.

In view of (5.26), the last inequality implies

$$
\frac{1}{2} \sum_{k=1}^{k_{n}} \varepsilon_{n k} \leq \sum_{k=1}^{k_{n}} \varepsilon_{n k, s} \leq c\left(\mu^{s}\right)<\infty, \quad n \geq n_{0} .
$$


Now we return to (5.2) and (5.3). Since $Q_{\mu^{(n)}}(z)=Q_{\widehat{\mu}_{n}}\left(e^{i\left(b_{n}-a_{n}\right)} z\right)$, where $b_{n}=$ $\sum_{k=1}^{k_{n}} a_{n k}$, we see that $Q_{\mu^{(n)}}(z)=Q_{\widehat{\mu}_{n k}}\left(Z_{n k}\left(e^{i\left(b_{n}-a_{n}\right)} z\right), z \in \mathbb{D}, k=1, \ldots, k_{n}\right.$. Since $Q_{\widehat{\mu}_{n k}}(z)$ tend to $z$ and $Q_{\widehat{\mu}_{n k}}\left(Z_{n k}\left(e^{i\left(b_{n}-a_{n}\right)} z\right)\right)$ tend to $Q_{\mu}(z)$ as $n \rightarrow \infty$ uniformly in $k=1, \ldots, k_{n}$ and on every compact set in $\mathbb{D}$, we obtain that $\left\{Z_{n k}\left(e^{i\left(b_{n}-a_{n}\right)} z\right)\right\}_{n=1}^{\infty}$ converges uniformly on every compact set in $\mathbb{D}$ to some function $Z(z) \in \mathcal{S}_{*}$. Using relations (5.2) and (5.3) with $e^{i\left(b_{n}-a_{n}\right)} z$ replacing $z$ and taking into account that the measures $\mu_{n 1}, \ldots, \mu_{n k_{n}}$ are infinitesimal and the upper bound (5.29) holds, we can repeat the arguments which we used for the proof of (5.18). Thus we arrive at the relation, for $z \in \mathbb{D}$,

$$
\begin{aligned}
& \frac{Q_{\widehat{\mu}_{n k}}\left(Z_{n 1}\left(e^{i\left(b_{n}-a_{n}\right)} z\right)\right)}{e^{i\left(b_{n}-a_{n}\right)} z} \\
= & \exp \left\{i \int_{[-\pi, \pi)} \frac{\sin y}{1-\cos y} \nu_{n}(d y)-\int_{[-\pi, \pi)} \frac{1+Z_{n 1}\left(e^{i\left(b_{n}-a_{n}\right)} z\right) e^{i y}}{1-Z_{n 1}\left(e^{i\left(b_{n}-a_{n}\right)} z\right) e^{i y}} \nu_{n}(d y)+\widehat{\rho}_{n}(z)\right\},
\end{aligned}
$$

where $\widehat{\rho}_{n}(z)$ is an analytic function in $\mathbb{D}$ and $\widehat{\rho}_{n}(z) \rightarrow 0$ on every compact set in $\mathbb{D}$. Denote $d_{n}:=\int_{[-\pi, \pi)} \frac{\sin y}{1-\cos y} \nu_{n}(d y)$.

By (5.29), the sequence $\left\{\nu_{n}\right\}$ is tight in the weak topology. Therefore there exists a subsequence $\left\{n^{\prime}\right\}$ such that $\left\{\nu_{n^{\prime}}\right\}$ converges to some finite nonnegative measure $\nu$ in the weak topology. Thus, we can conclude from (5.30) that $e^{i\left(b_{n^{\prime}}+d_{n^{\prime}}-a_{n^{\prime}}\right)} \rightarrow e^{i \alpha}$ as $n^{\prime} \rightarrow \infty$, where $\alpha \in[-\pi, \pi)$, and that the following relation holds

$$
\frac{Z(z)}{z}=\exp \left\{i \alpha-\int_{[-\pi, \pi)} \frac{1+Z(z) e^{i y}}{1-Z(z) e^{i y}} \nu(d y)\right\}, \quad z \in \mathbb{D} .
$$

We shall show that $\left\{\nu_{n}\right\}$ converges to a finite nonnegative measure $\nu$ in the weak topology. Assume to the contrary that there exists a subsequence $\left\{n^{\prime \prime}\right\}$ such that $\left\{\nu_{n^{\prime \prime}}\right\}$ converges in the weak topology to some finite nonnegative measure $\nu_{1} \not \equiv \nu$. Then $e^{i\left(b_{n^{\prime \prime}}+d_{n^{\prime \prime}}-a_{n^{\prime \prime}}\right)} \rightarrow e^{i \alpha_{1}}$ as $n^{\prime \prime} \rightarrow \infty$, where $\alpha_{1} \in[-\pi, \pi)$, and (5.31) holds with $\alpha_{1} \in(-\pi, \pi]$, replacing $\alpha$ and $\nu_{1}$ replacing $\nu$. We then deduce from (5.31) with old and new parameters

$$
\exp \left\{i \alpha-\int_{[-\pi, \pi)} \frac{1+z e^{i y}}{1-z e^{i y}} \nu(d y)\right\}=\exp \left\{i \alpha_{1}-\int_{[-\pi, \pi)} \frac{1+z e^{i y}}{1-z e^{i y}} \nu_{1}(d y)\right\}, \quad z \in \mathbb{D} \text {. }
$$

We see from (5.32) that

$$
\Re \int_{[-\pi, \pi)} \frac{1+z e^{i y}}{1-e^{i y}} \nu(d u)=\Re \int_{[-\pi, \pi)} \frac{1+z e^{i y}}{1-z e^{i y}} \nu_{1}(d y), \quad z \in \mathbb{D} .
$$

Applying the Stieltjes-Perron inversion formula (see Section 3), we obtain that $\nu=\nu_{1}$. Finally we get $\alpha=\alpha_{1}$.

Since $\left\{\nu_{n}\right\}$ converges to the measure $\nu$ in the weak topology, we obtain from (5.30) that $e^{i\left(b_{n}+d_{n}-a_{n}\right)} \rightarrow e^{i \alpha}$ as $n \rightarrow \infty$. 
Thus a necessity of the assumptions of the theorem is proved and Theorem 2.3 is completely proved.

Now we shall prove Theorem 2.4.

Proof. At first we shall prove the first statement of the theorem. Assume that the sequence $\mu_{n 1} \otimes \cdots \otimes \mu_{n k_{n}}$ converges weakly to a p-measure $\mu^{\bigotimes}$ such that $\Sigma_{\mu^{\otimes}}=[\alpha, \nu]$. By Theorem 2.3, we have $\nu_{n} \rightarrow \nu$, where $\nu_{n}$ is defined by the formula (2.17), and $\alpha_{n}=\alpha+o(1), \bmod 2 \pi$, where $\alpha_{n}$ is defined by the formula (2.18). Let us show that the sequence $\mu_{n 1} * \cdots * \mu_{n k_{n}}$ converges weakly to the p-measure $\mu^{*}$ such that $q_{\mu^{*}}=$ $\langle\alpha, \nu\rangle$. Consider the sequence $\left\{\mu_{n}\right\}$ of $*$-infinitely divisible measures with representations $\left\langle\alpha_{n}, \nu_{n}\right\rangle$. By Theorem 3.10, we have $\mu_{n} \rightarrow \mu$ weakly as $n \rightarrow \infty$, where $\mu$ is $*-$ infinitely divisible measure with a representation $\langle\alpha, \nu\rangle$. On the other hand we see that $\mu_{n}=x_{n} * e\left(\mu_{n 1} * x_{n 1}\right) * \cdots * e\left(\mu_{n k_{n}} * x_{n k_{n}}\right)$, where $x_{n}=-\left(x_{n 1}+\cdots+x_{n k_{n}}\right)$ and $x_{n k}$ are defined by (3.16). By Theorem 3.9, we obtain the first statement of the theorem.

As for the second statement of the theorem, consider p-measures $e\left(\nu_{j}\right), j=1,2$, where $\nu_{j}, j=1,2$, are measures from Proposition 3.11. Denote by $\mu_{n k}, n \geq 1, k=1, \ldots, n$, the p-measures $e\left(\nu_{1} / n\right)$. It is easy to see that $\bar{\mu}_{n k}=e\left(\nu_{2} / n\right)$ and $\mu_{n 1} * \cdots * \mu_{n n}=$ $\bar{\mu}_{n 1} * \cdots * \bar{\mu}_{n n}=e\left(\nu_{1}\right)$. The measures $\left\{\mu_{n k}, \bar{\mu}_{n k}\right\}$ are infinitesimal and the sequence $\left\{\mu_{n 1} * \cdots * \mu_{n n}, \bar{\mu}_{n 1} * \cdots * \bar{\mu}_{n n}\right\}$ obviously converges weakly to $e\left(\nu_{1}\right)=e\left(\nu_{2}\right)$. Let us show that the sequence $\left\{\delta_{a_{n}^{\prime}} \otimes \mu_{n 1} \otimes \cdots \otimes \mu_{n n}, \delta_{a_{n}^{\prime \prime}} \otimes \bar{\mu}_{n 1} \otimes \cdots \otimes \bar{\mu}_{n n}\right\}$ does not converge weakly to some p-measure in $\mathcal{M}_{*}$ for any $a_{n}^{\prime} \in \mathbb{T}, a_{n}^{\prime \prime} \in \mathbb{T}$. Assume to the contrary that there exists a sequence $a_{n}^{\prime} \in \mathbb{T}, a_{n}^{\prime \prime} \in \mathbb{T}$ such that the previous sequence of p-measures converge weakly to some p-measure in $\mathcal{M}_{*}$. Then, by Theorem 2.3, for every Borel set $S \subseteq[-\pi, \pi)$,

$$
n \int_{S}(1-\cos y) \widehat{\mu}_{n k}(d y) \rightarrow \nu(S), \quad n \int_{S}(1-\cos y) \widehat{\bar{\mu}}_{n k}(d y) \rightarrow \nu(S), \quad n \rightarrow \infty,
$$

where $\nu$ is some finite nonnegative measure and $\widehat{\mu}_{n k}=\mu_{n k} \otimes \delta_{a_{n k}}, \widehat{\bar{\mu}}_{n k}=\bar{\mu}_{n k} \otimes \delta_{-a_{n k}}$ with $a_{n k}=-\int_{[-\pi, \pi)} \sin y \mu_{n k}(d y)$. Since, by the definition of $\mu_{n k}$,

$$
\begin{aligned}
& \mu_{n k}=e^{-\nu_{1}([-\pi, \pi)) / n}\left(\delta_{0}+\frac{\nu_{1}}{n}+\frac{1}{2 !} \frac{\nu_{1} * \nu_{1}}{n^{2}}+\ldots\right), \\
& \bar{\mu}_{n k}=e^{-\nu_{2}([-\pi, \pi)) / n}\left(\delta_{0}+\frac{\nu_{2}}{n}+\frac{1}{2 !} \frac{\nu_{2} * \nu_{2}}{n^{2}}+\ldots\right),
\end{aligned}
$$

we note from (5.33) that

$$
\nu_{1}((x, \pi))=\nu((x, \pi)) \quad \text { and } \quad \nu_{2}((x, \pi))=\nu((x, \pi))
$$

for every fixed $x \in(0, \pi)$, a contradiction. Thus the theorem is proved completely.

\section{REFERENCES}

[1] Akhiezer, N. I. The classical moment problem and some related questions in analysis. Hafner, New York (1965). 
[2] Akhiezer, N. I. and Glazman, I. M. Theory of Linear Operators in Hilbert Space. Ungar, New York (1963).

[3] Barndorff-Nielsen, O. E. and Thorbjørnsen, S. Selfdecomposability and Lévy processes in free probability. Bernoulli, 8, 323-366 (2002).

[4] Belinschi, S. T. Complex analysis methods on noncommutative probability. arXiv:math.OA/0603104 v 1 (2006).

[5] Bercovici, H., and Voiculescu, D. Lévy-Hinčin type theorems for multiplicative and additive free convolution. Pacific journal of mathematics, 153, 217-248 (1992).

[6] Bercovici, H., and Voiculescu, D. Free convolution of measures with unbounded support. Indiana Univ. Math. J., 42, 733-773 (1993).

[7] Bercovici, H., and Pata, V. Stable laws and domains of attraction in free probability theory. Annals of Math., 149, 1023-1060, (1999).

[8] Bercovici, H., and Pata, V. Limit laws for products of free and independent random variables. Studia Mathematica, 141, 43-52 (2000).

[9] Biane, Ph. Processes with free increments. Math. Z., 227, 143-174 (1998).

[10] Chistyakov, G. P. and Götze, F. The arithmetic of distributions in free probability theory. arXiv:math. $\mathrm{OA} / 0508245$ v 1 (2005).

[11] Chistyakov, G. P. and Götze, F. Limit theorems in free probability theory. I arXiv:math. OA/0602219 v 1 (2006).

[12] Gnedenko, B. V. and Kolmogorov, A. N. Limit distributions for sums of independent random variables. Addison-Wesley Publishing Company, (1968).

[13] Loéve, M. Probability theory. VNR, New York, (1963).

[14] Parthasarathy, K. R. Probability measures on metric spaces. Academic Press, New York and London, (1967).

[15] Sazonov, V. V. and Tutubalin V. N. Probability distributions on topological groups Theory Probab. and its Applic., 11, N 1, 1-45 (1966).

[16] Voiculescu, D.V. Multiplication of certain noncommuting random variavles. J. Operator Theory, 18, 223-235 (1987).

[17] Voiculesku, D., Dykema, K., and Nica, A. Free random variables. CRM Monograph Series, No 1, A.M.S., Providence, RI (1992).

[18] Voiculescu, D.V. The analogues of entropy and Fisher's information mesure in free probability theory. I. Comm. Math. Phys., 155, 71-92 (1993).

[19] Voiculescu, D.V. The coalgebra of the free difference quotient and free probability. Int. Math. Res. Not., no. 2, 79-106 (2000).

[20] Voiculescu, D.V. Analytic subordination consequences of free Markovianity. Indiana Univ. Math. J., 51, 1161-1166 (2002)

\section{Gennadil Chistyakov}

Institute FOR LOW Temperature Physics AND EngineERING

National Academy of Sciences of Ukraine

47 LENIN Ave.

61103 KHARKOV

UKRAINE 
E-mail address: chistyakov@ilt.kharkov.ua, chistyak@math.bielefeld.de

FrIEDRICH GÖTZE

FAKULTÄT FÜR MATHEMATIK

UNIVERSITÄT BIELEFELD

POSTFACH 100131

33501 BIELEFELD 1

Germany

E-mail address: goetze@mathematik.uni-bielefeld.de 